\title{
Facile Uncatalyzed Mukaiyama Aldol Reactions: An Ab Initio Study of the Effects of Substituents
}

\author{
Chiong Teck Wong and Ming Wah Wong* \\ Department of Chemistry, National University of Singapore, \\ 3 Science Drive 3, Singapore 117543 \\ chmwmw@nus.edu.sg
}

\section{Table of Contents (23 pages)}

Page S2: $\quad$ G3(MP2) $\mathrm{E}_{0}$ Total Energies for the Reactants (Table S1)

Pages S3-S4: G3(MP2) E 0 Total Energies for the Transition States and Products (Table S2)

Page S5: Calculated B3LYP/6-31G* Barriers and Enthalpies Gas Phase $(\varepsilon=1)$ and a Polar Medium $(\varepsilon=40)$ (Table S3)

Page S6: $\quad$ Calculated HOMO and LUMO Energies of the Reactants (Table S4)

Page S7: $\quad$ Optimized Bond Lengths of the Reactants (Table S5)

Page S8: $\quad$ Optimized Bond Lengths of the Transition States (Table S6)

Page S9: $\quad$ NBO Atomic Charges of the Reactants (Table S7)

Pages S10-23: Cartesian Coordinates of Optimized MP2(full)/6-31G* Geometries of Reactants, Transition States, Intermediate and Products (Table S8) 
Table S1. G3(MP2) $E_{0}$ Total Energies (hartree) for the Reactants

\begin{tabular}{|cc|c|}
\hline \multirow{2}{*}{$\mathrm{R}_{\mathrm{a}}$} & $\mathrm{R}_{\mathrm{b}}$ & G3(MP2) energy \\
\cline { 3 - 3 } & & Aldehyde \\
\hline \hline $\mathrm{H}$ & $\mathrm{H}$ & -114.35304 \\
$\mathrm{CF}_{3}$ & $\mathrm{H}$ & -451.09233 \\
$\mathrm{CH}_{3}$ & $\mathrm{H}$ & -153.59969 \\
$\mathrm{CH}_{3}$ & $\mathrm{CH}_{3}$ & -192.84511 \\
$\mathrm{CHO}$ & $\mathrm{H}$ & -227.53572 \\
$\mathrm{~F}$ & $\mathrm{H}$ & -213.54323 \\
$\mathrm{NH}_{2}$ & $\mathrm{H}$ & -169.66756 \\
$\mathrm{CH}=\mathrm{CH}_{2}$ & $\mathrm{H}$ & -191.62272 \\
$\mathrm{C}_{6} \mathrm{H}_{5}$ & $\mathrm{H}$ & -345.01972 \\
$\mathrm{COOCH}_{3}$ & $\mathrm{H}$ & -341.93451 \\
\hline \hline & oxetane & -558.24562 \\
\hline
\end{tabular}

\begin{tabular}{|cccc|c|}
\hline & & & & G3(MP2) energy \\
\cline { 5 - 5 } $\mathrm{R}_{\mathrm{c}}$ & $\mathrm{R}_{\mathrm{d}}$ & $\mathrm{R}_{\mathrm{e}}$ & $\mathrm{O}-\mathrm{R}$ & Ether \\
\hline \hline $\mathrm{H}$ & $\mathrm{H}$ & $\mathrm{H}$ & $\mathrm{SiH}_{3}$ & -443.87539 \\
$\mathrm{CH}_{3}$ & $\mathrm{H}$ & $\mathrm{H}$ & $\mathrm{SiH}_{3}$ & -483.11871 \\
$\mathrm{CHO}$ & $\mathrm{H}$ & $\mathrm{H}$ & $\mathrm{SiH}_{3}$ & -557.06264 \\
$\mathrm{~F}$ & $\mathrm{H}$ & $\mathrm{H}$ & $\mathrm{SiH}_{3}$ & -543.04370 \\
$\mathrm{NH}_{2}$ & $\mathrm{H}$ & $\mathrm{H}$ & $\mathrm{SiH}_{3}$ & -499.16499 \\
$\mathrm{OH}$ & $\mathrm{H}$ & $\mathrm{H}$ & $\mathrm{SiH}_{3}$ & -519.03351 \\
$\mathrm{SH}$ & $\mathrm{H}$ & $\mathrm{H}$ & $\mathrm{SiH}_{3}$ & -841.63504 \\
$\mathrm{H}$ & $\mathrm{NH}$ & $\mathrm{H}$ & $\mathrm{SiH}_{3}$ & -499.14815 \\
$\mathrm{H}$ & $\mathrm{H}$ & $\mathrm{NH}$ & $\mathrm{SiH}_{3}$ & -499.15129 \\
$\mathrm{H}$ & $\mathrm{H}$ & $\mathrm{H}$ & $\mathrm{Si}_{2}\left(\mathrm{CH}_{3}\right)_{3}$ & -561.63585 \\
$\mathrm{H}$ & $\mathrm{H}$ & $\mathrm{H}$ & $\mathrm{Si}\left(\mathrm{C}_{3} \mathrm{H}_{6}\right)\left(\mathrm{CH}_{3}\right)$ & -599.64141 \\
$\mathrm{H}$ & $\mathrm{H}$ & $\mathrm{H}$ & $\mathrm{SiF}_{3}$ & -741.61284 \\
$\mathrm{H}$ & $\mathrm{H}$ & $\mathrm{H}$ & $\mathrm{SiCl}_{3}$ & -1821.53387 \\
\hline
\end{tabular}


Table S2. G3(MP2) E $E_{0}$ Total Energies (hartree) for the Transition States and Products

\begin{tabular}{|cccccc|cc|}
\hline \multirow{2}{*}{$\mathrm{R}_{\mathrm{a}}$} & $\mathrm{R}_{\mathrm{b}}$ & $\mathrm{R}_{\mathrm{c}}$ & $\mathrm{R}_{\mathrm{d}}$ & $\mathrm{R}_{\mathrm{e}}$ & $\mathrm{O}-\mathrm{R}$ & \multicolumn{2}{|c|}{ G3(MP2) Energy } \\
\hline \hline $\mathrm{H}$ & & & & & & Transition State & Product \\
$\mathrm{CF}_{3}$ & $\mathrm{H}$ & $\mathrm{H}$ & $\mathrm{H}$ & $\mathrm{H}$ & $\mathrm{SiH}_{3}$ & -558.19925 & -558.26459 \\
$\mathrm{CH}_{3}$ & $\mathrm{H}$ & $\mathrm{H}$ & $\mathrm{H}$ & $\mathrm{H}$ & $\mathrm{SiH}_{3}$ & -894.94683 & -895.01235 \\
$\mathrm{CH}_{3}$ & $\mathrm{CH}$ & $\mathrm{H}$ & $\mathrm{H}$ & $\mathrm{H}$ & $\mathrm{SiH}_{3}$ & -597.44349 & -597.50451 \\
$\mathrm{CHO}$ & $\mathrm{H}$ & $\mathrm{H}$ & $\mathrm{H}$ & $\mathrm{H}$ & $\mathrm{SiH}_{3}$ & -636.68515 & -636.74567 \\
$\mathrm{~F}$ & $\mathrm{H}$ & $\mathrm{H}$ & $\mathrm{H}$ & $\mathrm{H}$ & $\mathrm{SiH}_{3}$ & -671.38680 & -671.44930 \\
$\mathrm{NH} \mathrm{H}_{2}$ & $\mathrm{H}$ & $\mathrm{H}$ & $\mathrm{H}$ & $\mathrm{H}$ & $\mathrm{SiH}_{3}$ & -657.37931 & -657.43937 \\
$\mathrm{CH}=\mathrm{CH}$ & $\mathrm{H}$ & $\mathrm{H}$ & $\mathrm{H}$ & $\mathrm{H}$ & $\mathrm{SiH}_{3}$ & -613.49290 & -613.54747 \\
$\mathrm{C}_{6} \mathrm{H}_{5}$ & $\mathrm{H}$ & $\mathrm{H}$ & $\mathrm{H}$ & $\mathrm{H}$ & $\mathrm{SiH}_{3}$ & -635.46540 & -635.52473 \\
$\mathrm{COOCH}$ & $\mathrm{H}$ & $\mathrm{H}$ & $\mathrm{H}$ & $\mathrm{H}$ & $\mathrm{SiH}_{3}$ & -788.86536 & -788.92278 \\
$\mathrm{H}$ & $\mathrm{H}$ & $\mathrm{CH}$ & $\mathrm{H}$ & $\mathrm{H}$ & $\mathrm{SiH}_{3}$ & -785.78670 & -785.85121 \\
$\mathrm{H}$ & $\mathrm{H}$ & $\mathrm{CHO}$ & $\mathrm{H}$ & $\mathrm{H}$ & $\mathrm{SiH}_{3}$ & -597.44747 & -597.51091 \\
$\mathrm{H}$ & $\mathrm{H}$ & $\mathrm{F}$ & $\mathrm{H}$ & $\mathrm{H}$ & $\mathrm{SiH}_{3}$ & -671.38559 & -671.44978 \\
$\mathrm{H}$ & $\mathrm{H}$ & $\mathrm{NH}$ & $\mathrm{H}$ & $\mathrm{H}$ & $\mathrm{SiH}_{3}$ & -657.37606 & -657.45555 \\
$\mathrm{H}$ & $\mathrm{H}$ & $\mathrm{OH}$ & $\mathrm{H}$ & $\mathrm{H}$ & $\mathrm{SiH}_{3}$ & -613.50657 & -613.57973 \\
$\mathrm{H}$ & $\mathrm{H}$ & $\mathrm{SH}$ & $\mathrm{H}$ & $\mathrm{H}$ & $\mathrm{SiH}_{3}$ & -633.37215 & -633.44791 \\
$\mathrm{H}$ & $\mathrm{H}$ & $\mathrm{H}$ & $\mathrm{NH}$ & $\mathrm{H}$ & $\mathrm{SiH}_{3}$ & -955.96884 & -956.03871 \\
$\mathrm{H}$ & $\mathrm{H}$ & $\mathrm{H}$ & $\mathrm{H}$ & $\mathrm{NH}$ & $\mathrm{SiH}_{3}$ & -613.47445 & -613.53899 \\
$\mathrm{CHO}$ & $\mathrm{H}$ & $\mathrm{NH}$ & $\mathrm{H}$ & $\mathrm{H}$ & $\mathrm{SiH}_{3}$ & -613.47294 & -613.54374 \\
& & & & -726.69628 & -726.76384
\end{tabular}




\begin{tabular}{|cccccc|cc|}
$\mathrm{H}$ & $\mathrm{H}$ & $\mathrm{H}$ & $\mathrm{H}$ & $\mathrm{H}$ & $\mathrm{Si}\left(\mathrm{CH}_{3}\right)_{3}$ & -675.95201 & -676.02639 \\
$\mathrm{H}$ & $\mathrm{H}$ & $\mathrm{H}$ & $\mathrm{H}$ & $\mathrm{H}$ & $\mathrm{Si}\left(\mathrm{C}_{3} \mathrm{H}_{6}\right)\left(\mathrm{CH}_{3}\right)$ & -713.96953 & -714.03099 \\
$\mathrm{H}$ & $\mathrm{H}$ & $\mathrm{H}$ & $\mathrm{H}$ & $\mathrm{H}$ & $\mathrm{SiF}_{3}$ & -855.94800 & -856.00541 \\
$\mathrm{H}$ & $\mathrm{H}$ & $\mathrm{H}$ & $\mathrm{H}$ & $\mathrm{H}$ & $\mathrm{SiCl}_{3}$ & -1935.86219 & -1935.92721 \\
\hline \hline & & & & $\mathbf{T S}_{\mathbf{C}}$ & -558.19925 & \\
& & & & & $\mathbf{T S}_{\mathbf{S} 1}$ & -558.15172 & \\
& & & & & $\mathbf{T S}_{\mathbf{S} 2}$ & -558.20204 & \\
\hline
\end{tabular}


Table S3. Calculated B3LYP/6-31G* Barriers and Enthalpies $\left(\mathrm{kJ} \mathrm{mol}^{-1}\right)$ Gas Phase $(\varepsilon=1)$ and a Polar Medium $(\varepsilon=40)$

\begin{tabular}{|c|c|c|c|c|c|c|c|c|c|}
\hline \multirow{2}{*}{$\mathrm{R}_{\mathrm{a}}$} & \multirow{2}{*}{$\mathrm{R}_{\mathrm{b}}$} & \multirow{2}{*}{$\mathrm{R}_{\mathrm{c}}$} & \multirow{2}{*}{$\mathrm{R}_{\mathrm{d}}$} & \multirow{2}{*}{$\mathrm{R}_{\mathrm{e}}$} & \multirow{2}{*}{$\mathrm{O}-\mathrm{R}$} & \multicolumn{2}{|c|}{ Barrier } & \multicolumn{2}{|c|}{ Enthalpy } \\
\hline & & & & & & $\varepsilon=1$ & $\varepsilon=40$ & $\varepsilon=1$ & $\varepsilon=40$ \\
\hline $\mathrm{H}$ & $\mathrm{H}$ & $\mathrm{H}$ & $\mathrm{H}$ & $\mathrm{H}$ & $\mathrm{SiH}_{3}$ & 63.9 & 59.5 & -96.8 & -94.9 \\
\hline $\mathrm{CF}_{3}$ & $\mathrm{H}$ & $\mathrm{H}$ & $\mathrm{H}$ & $\mathrm{H}$ & $\mathrm{SiH}_{3}$ & 51.4 & 49.9 & -110.5 & -110.7 \\
\hline $\mathrm{CH}_{3}$ & $\mathrm{H}$ & $\mathrm{H}$ & $\mathrm{H}$ & $\mathrm{H}$ & $\mathrm{SiH}_{3}$ & 79.7 & 75.5 & -66.4 & -63.5 \\
\hline $\mathrm{CH}_{3}$ & $\mathrm{CH}_{3}$ & $\mathrm{H}$ & $\mathrm{H}$ & $\mathrm{H}$ & $\mathrm{SiH}_{3}$ & 101.5 & 96.9 & -42.0 & -40.8 \\
\hline $\mathrm{CHO}$ & $\mathrm{H}$ & $\mathrm{H}$ & $\mathrm{H}$ & $\mathrm{H}$ & $\mathrm{SiH}_{3}$ & 57.9 & 55.3 & -91.5 & -96.4 \\
\hline $\mathrm{F}$ & $\mathrm{H}$ & $\mathrm{H}$ & $\mathrm{H}$ & $\mathrm{H}$ & $\mathrm{SiH}_{3}$ & 93.6 & 92.2 & -57.5 & -57.2 \\
\hline $\mathrm{NH}_{2}$ & $\mathrm{H}$ & $\mathrm{H}$ & $\mathrm{H}$ & $\mathrm{H}$ & $\mathrm{SiH}_{3}$ & 127.2 & 124.8 & 3.9 & 8.8 \\
\hline $\mathrm{CH}=\mathrm{CH}_{2}$ & $\mathrm{H}$ & $\mathrm{H}$ & $\mathrm{H}$ & $\mathrm{H}$ & $\mathrm{SiH}_{3}$ & 85.5 & 82.3 & -50.2 & -50.3 \\
\hline $\mathrm{C}_{6} \mathrm{H}_{5}$ & $\mathrm{H}$ & $\mathrm{H}$ & $\mathrm{H}$ & $\mathrm{H}$ & $\mathrm{SiH}_{3}$ & 86.7 & 84.1 & -46.9 & -43.0 \\
\hline $\mathrm{COOCH}_{3}$ & $\mathrm{H}$ & $\mathrm{H}$ & $\mathrm{H}$ & $\mathrm{H}$ & $\mathrm{SiH}_{3}$ & 60.6 & 58.5 & -94.1 & -99.8 \\
\hline $\mathrm{H}$ & $\mathrm{H}$ & $\mathrm{CH}_{3}$ & $\mathrm{H}$ & $\mathrm{H}$ & $\mathrm{SiH}_{3}$ & 53.7 & 51.0 & -103.0 & -101.0 \\
\hline $\mathrm{H}$ & $\mathrm{H}$ & $\mathrm{CHO}$ & $\mathrm{H}$ & $\mathrm{H}$ & $\mathrm{SiH}_{3}$ & 71.1 & 78.8 & -84.8 & -76.0 \\
\hline $\mathrm{H}$ & $\mathrm{H}$ & $\mathrm{F}$ & $\mathrm{H}$ & $\mathrm{H}$ & $\mathrm{SiH}_{3}$ & 41.0 & 41.7 & -154.9 & -153.3 \\
\hline $\mathrm{H}$ & $\mathrm{H}$ & $\mathrm{NH}_{2}$ & $\mathrm{H}$ & $\mathrm{H}$ & $\mathrm{SiH}_{3}$ & 19.9 & 18.5 & -153.8 & -154.8 \\
\hline $\mathrm{H}$ & $\mathrm{H}$ & $\mathrm{OH}$ & $\mathrm{H}$ & $\mathrm{H}$ & $\mathrm{SiH}_{3}$ & 24.9 & 30.0 & -165.2 & -159.9 \\
\hline $\mathrm{H}$ & $\mathrm{H}$ & SH & $\mathrm{H}$ & $\mathrm{H}$ & $\mathrm{SiH}_{3}$ & 41.2 & 44.7 & -138.9 & -133.8 \\
\hline $\mathrm{CHO}$ & $\mathrm{H}$ & $\mathrm{NH}_{2}$ & $\mathrm{H}$ & $\mathrm{H}$ & $\mathrm{SiH}_{3}$ & 7.1 & 1.9 & -159.3 & -160.3 \\
\hline $\mathrm{H}$ & $\mathrm{H}$ & $\mathrm{H}$ & $\mathrm{H}$ & $\mathrm{H}$ & $\mathrm{Si}\left(\mathrm{CH}_{3}\right)_{3}$ & 95.0 & 97.0 & -98.0 & -99.3 \\
\hline $\mathrm{H}$ & $\mathrm{H}$ & $\mathrm{H}$ & $\mathrm{H}$ & $\mathrm{H}$ & $\mathrm{Si}\left(\mathrm{C}_{3} \mathrm{H}_{6}\right)\left(\mathrm{CH}_{3}\right)$ & 65.6 & 67.7 & -99.5 & -98.3 \\
\hline $\mathrm{H}$ & $\mathrm{H}$ & $\mathrm{H}$ & $\mathrm{H}$ & $\mathrm{H}$ & $\mathrm{GeH}_{3}$ & 49.2 & 49.6 & -95.7 & -93.2 \\
\hline
\end{tabular}


Table S4. Calculated HOMO and LUMO Energies (hartree) of the Reactants

\begin{tabular}{|cc|cccc|}
\hline \multicolumn{3}{|c|}{ Aldehyde } & \multicolumn{4}{c|}{ MP2(full)/6-31G* energy } \\
\hline $\mathrm{R}_{\mathrm{a}}$ & $\mathrm{R}_{\mathrm{b}}$ & $\mathrm{HOMO}$ & $\mathrm{LUMO}$ & $\Delta \mathrm{E}_{\mathrm{AS}}^{\mathrm{a}}$ & $\Delta \mathrm{E}_{\mathrm{SA}}{ }^{\mathrm{b}}$ \\
\hline \hline $\mathrm{H}$ & $\mathrm{H}$ & -0.43937 & 0.13433 & 0.57797 & 0.48417 \\
$\mathrm{CF}_{3}$ & $\mathrm{H}$ & -0.48066 & 0.09335 & 0.61926 & 0.44319 \\
$\mathrm{CH}_{3}$ & $\mathrm{H}$ & -0.42367 & 0.15152 & 0.56227 & 0.50136 \\
$\mathrm{CH}_{3}$ & $\mathrm{CH}_{3}$ & -0.41122 & 0.15749 & 0.54982 & 0.50733 \\
$\mathrm{CHO}_{\mathrm{F}}$ & $\mathrm{H}$ & -0.44056 & 0.04463 & 0.57916 & 0.39447 \\
$\mathrm{NH}_{2}$ & $\mathrm{H}$ & -0.50656 & 0.14518 & 0.64516 & 0.49502 \\
$\mathrm{CH}_{\mathrm{CH}}$ & $\mathrm{H}$ & -0.41321 & 0.19787 & 0.55181 & 0.54771 \\
$\mathrm{C}_{6} \mathrm{H}_{5}$ & $\mathrm{H}$ & -0.39378 & 0.08918 & 0.53238 & 0.43902 \\
$\mathrm{COOCH}_{3}$ & $\mathrm{H}$ & -0.34631 & 0.07842 & 0.48491 & 0.42826 \\
& $\mathrm{H}$ & -0.44552 & 0.06446 & 0.58412 & 0.41430 \\
Trihydrosilyl Enol Ether & -0.34984 & 0.13860 & & \\
\hline
\end{tabular}

\begin{tabular}{|ccc|cccc|}
\hline \multicolumn{3}{|c|}{ Trihydrosilyl Enol Ether } & \multicolumn{5}{c|}{ MP2(full)/6-31G* energy } \\
\hline $\mathrm{R}_{\mathrm{c}}$ & $\mathrm{R}_{\mathrm{d}}$ & $\mathrm{R}_{\mathrm{e}}$ & $\mathrm{HOMO}$ & LUMO & $\Delta \mathrm{E}_{\mathrm{AS}}{ }^{\mathrm{a}}$ & $\Delta \mathrm{E}_{\mathrm{SA}}{ }^{\mathrm{b}}$ \\
\hline \hline $\mathrm{H}$ & $\mathrm{H}$ & $\mathrm{H}$ & -0.34984 & 0.13860 & 0.57797 & 0.48417 \\
$\mathrm{CH}_{3}$ & $\mathrm{H}$ & $\mathrm{H}$ & -0.33984 & 0.14009 & 0.57946 & 0.47417 \\
$\mathrm{CHO}$ & $\mathrm{H}$ & $\mathrm{H}$ & -0.36450 & 0.08779 & 0.52716 & 0.49883 \\
$\mathrm{~F}$ & $\mathrm{H}$ & $\mathrm{H}$ & -0.35678 & 0.12329 & 0.56266 & 0.49111 \\
$\mathrm{NH}_{2}$ & $\mathrm{H}$ & $\mathrm{H}$ & -0.31435 & 0.13901 & 0.57838 & 0.44868 \\
$\mathrm{OH}$ & $\mathrm{H}$ & $\mathrm{H}$ & -0.33031 & 0.12694 & 0.56631 & 0.46464 \\
$\mathrm{SH}$ & $\mathrm{H}$ & $\mathrm{H}$ & -0.33404 & 0.13066 & 0.57003 & 0.46837 \\
& & & & & & \\
\hline
\end{tabular}

a $\Delta \mathrm{E}_{\mathrm{AS}}$ : Energy difference between LUMO(trihydrosilyl enol ether) and HOMO(aldehyde).

b $\Delta \mathrm{E}_{\mathrm{SA}}$ : Energy difference between LUMO(aldehyde) and HOMO(trihydrosilyl enol ether). 
Table S5. Optimized (MP2(full)/6-31G*) Bond Lengths (Á) of the Reactants

\begin{tabular}{|cc|c|}
\hline $\mathrm{R}_{\mathrm{a}}$ & $\mathrm{R}_{\mathrm{b}}$ & $\mathrm{C}_{\gamma}-\mathrm{O}_{\beta}$ \\
\hline \hline Aldehyde & & \\
$\mathrm{H}$ & $\mathrm{H}$ & 1.220 \\
$\mathrm{CF}_{3}$ & $\mathrm{H}$ & 1.215 \\
$\mathrm{CH}_{3}$ & $\mathrm{H}$ & 1.223 \\
$\mathrm{CH}_{3}$ & $\mathrm{CH}_{3}$ & 1.226 \\
$\mathrm{CHO}$ & $\mathrm{H}$ & 1.223 \\
$\mathrm{~F}$ & $\mathrm{H}$ & 1.194 \\
$\mathrm{NH}_{2}$ & $\mathrm{H}$ & 1.224 \\
$\mathrm{CH}_{2} \mathrm{CH}_{2}$ & $\mathrm{H}$ & 1.226 \\
$\mathrm{C}_{6} \mathrm{H}_{5}$ & $\mathrm{H}$ & 1.226 \\
$\mathrm{COOCH}_{3}$ & $\mathrm{H}$ & 1.219 \\
\hline
\end{tabular}

\begin{tabular}{|ccc|ccc|}
\hline $\mathrm{R}_{\mathrm{c}}$ & $\mathrm{R}_{\mathrm{d}}$ & $\mathrm{R}_{\mathrm{e}}$ & $\mathrm{Si}-\mathrm{O}_{\alpha}$ & $\mathrm{O}_{\alpha}-\mathrm{C}_{\alpha}$ & $\mathrm{C}_{\alpha}-\mathrm{C}_{\beta}$ \\
\hline \hline Ether & & & & & \\
$\mathrm{H}$ & $\mathrm{H}$ & $\mathrm{H}$ & 1.683 & 1.371 & 1.337 \\
$\mathrm{CH}_{3}$ & $\mathrm{H}$ & $\mathrm{H}$ & 1.681 & 1.380 & 1.340 \\
$\mathrm{CHO}$ & $\mathrm{H}$ & $\mathrm{H}$ & 1.688 & 1.371 & 1.342 \\
$\mathrm{~F}$ & $\mathrm{H}$ & $\mathrm{H}$ & 1.691 & 1.344 & 1.333 \\
$\mathrm{NH}_{2}$ & $\mathrm{H}$ & $\mathrm{H}$ & 1.685 & 1.376 & 1.342 \\
$\mathrm{OH}$ & $\mathrm{H}$ & $\mathrm{H}$ & 1.690 & 1.369 & 1.337 \\
$\mathrm{SH}$ & $\mathrm{H}$ & $\mathrm{H}$ & 1.690 & 1.370 & 1.341 \\
$\mathrm{H}$ & $\mathrm{NH}$ & $\mathrm{H}$ & 1.680 & 1.370 & 1.339 \\
$\mathrm{H}$ & $\mathrm{H}$ & $\mathrm{NH}_{2}$ & 1.702 & 1.356 & 1.339 \\
\hline
\end{tabular}


Table S6. Optimized (MP2(full)/6-31G*) Bond Lengths (Á) of the Transition States

\begin{tabular}{|c|c|c|c|c|c|c|c|c|c|c|}
\hline $\mathrm{R}_{\mathrm{a}}$ & $\mathrm{R}_{\mathrm{b}}$ & $\mathrm{R}_{\mathrm{c}}$ & $\mathrm{R}_{\mathrm{d}}$ & $\mathrm{R}_{\mathrm{e}}$ & $\mathrm{Si}-\mathrm{O}_{\alpha}$ & $\mathrm{O}_{\alpha}-\mathrm{C}_{\alpha}$ & $\mathrm{C}_{\alpha}-\mathrm{C}_{\beta}$ & $\mathrm{C}_{\beta}-\mathrm{C}_{\gamma}$ & $\mathrm{C}_{\gamma}-\mathrm{O}_{\beta}$ & $\mathrm{O}_{\beta}-\mathrm{Si}$ \\
\hline \multicolumn{11}{|c|}{ Transition States } \\
\hline $\mathrm{H}$ & $\mathrm{H}$ & $\mathrm{H}$ & $\mathrm{H}$ & $\mathrm{H}$ & 1.807 & 1.295 & 1.378 & 2.076 & 1.279 & 2.004 \\
\hline $\mathrm{CF}_{3}$ & $\mathrm{H}$ & $\mathrm{H}$ & $\mathrm{H}$ & $\mathrm{H}$ & 1.778 & 1.297 & 1.379 & 2.062 & 1.269 & 2.146 \\
\hline $\mathrm{CH}_{3}$ & $\mathrm{H}$ & $\mathrm{H}$ & $\mathrm{H}$ & $\mathrm{H}$ & 1.822 & 1.292 & 1.381 & 2.062 & 1.287 & 1.963 \\
\hline $\mathrm{CH}_{3}$ & $\mathrm{CH}_{3}$ & $\mathrm{H}$ & $\mathrm{H}$ & $\mathrm{H}$ & 1.810 & 1.294 & 1.379 & 2.056 & 1.291 & 1.992 \\
\hline $\mathrm{CHO}$ & $\mathrm{H}$ & $\mathrm{H}$ & $\mathrm{H}$ & $\mathrm{H}$ & 1.816 & 1.290 & 1.384 & 2.054 & 1.284 & 2.014 \\
\hline $\mathrm{F}$ & $\mathrm{H}$ & $\mathrm{H}$ & $\mathrm{H}$ & $\mathrm{H}$ & 1.790 & 1.289 & 1.387 & 1.881 & 1.257 & 2.141 \\
\hline $\mathrm{NH}_{2}$ & $\mathrm{H}$ & $\mathrm{H}$ & $\mathrm{H}$ & $\mathrm{H}$ & 1.902 & 1.278 & 1.397 & 1.923 & 1.314 & 1.866 \\
\hline $\mathrm{C}_{2} \mathrm{H}_{3}$ & $\mathrm{H}$ & $\mathrm{H}$ & $\mathrm{H}$ & $\mathrm{H}$ & 1.838 & 1.289 & 1.385 & 2.034 & 1.295 & 1.938 \\
\hline $\mathrm{C}_{6} \mathrm{H}_{5}$ & $\mathrm{H}$ & $\mathrm{H}$ & $\mathrm{H}$ & $\mathrm{H}$ & 1.833 & 1.290 & 1.384 & 2.034 & 1.294 & 1.949 \\
\hline $\mathrm{COOCH}_{3}$ & $\mathrm{H}$ & $\mathrm{H}$ & $\mathrm{H}$ & $\mathrm{H}$ & 1.809 & 1.292 & 1.382 & 2.056 & 1.278 & 2.024 \\
\hline $\mathrm{H}$ & $\mathrm{H}$ & $\mathrm{CH}_{3}$ & $\mathrm{H}$ & $\mathrm{H}$ & 1.787 & 1.305 & 1.379 & 2.092 & 1.276 & 2.041 \\
\hline $\mathrm{H}$ & $\mathrm{H}$ & $\mathrm{CHO}$ & $\mathrm{H}$ & $\mathrm{H}$ & 1.823 & 1.297 & 1.382 & 2.036 & 1.284 & 1.988 \\
\hline $\mathrm{H}$ & $\mathrm{H}$ & F & $\mathrm{H}$ & $\mathrm{H}$ & 1.800 & 1.279 & 1.370 & 2.181 & 1.268 & 2.059 \\
\hline $\mathrm{H}$ & $\mathrm{H}$ & $\mathrm{NH}_{2}$ & $\mathrm{H}$ & $\mathrm{H}$ & 1.739 & 1.322 & 1.375 & 2.242 & 1.257 & 2.234 \\
\hline $\mathrm{H}$ & $\mathrm{H}$ & $\mathrm{OH}$ & $\mathrm{H}$ & $\mathrm{H}$ & 1.766 & 1.310 & 1.368 & 2.237 & 1.261 & 2.152 \\
\hline $\mathrm{H}$ & $\mathrm{H}$ & $\mathrm{SH}$ & $\mathrm{H}$ & $\mathrm{H}$ & 1.784 & 1.305 & 1.375 & 2.167 & 1.267 & 2.076 \\
\hline $\mathrm{H}$ & $\mathrm{H}$ & $\mathrm{H}$ & $\mathrm{NH}_{2}$ & $\mathrm{H}$ & 1.797 & 1.297 & 1.379 & 2.081 & 1.276 & 2.029 \\
\hline $\mathrm{H}$ & $\mathrm{H}$ & $\mathrm{H}$ & $\mathrm{H}$ & $\mathrm{NH}_{2}$ & 1.948 & 1.285 & 1.391 & 2.045 & 1.309 & 1.847 \\
\hline $\mathrm{CHO}$ & $\mathrm{H}$ & $\mathrm{NH}_{2}$ & $\mathrm{H}$ & $\mathrm{H}$ & 1.743 & 1.318 & 1.380 & 2.231 & 1.259 & 2.267 \\
\hline
\end{tabular}


Table S7. NBO Atomic Charges of the Reactants ${ }^{\mathrm{a}}$

\begin{tabular}{|c|c|c|c|c|}
\hline $\mathrm{R}_{\mathrm{a}}$ & $\mathrm{R}_{\mathrm{b}}$ & $\mathrm{C}_{\gamma}$ & $\mathrm{O}_{\beta}$ & "R $\mathrm{R}_{\mathrm{a}}$ \\
\hline $\mathrm{H}$ & $\mathrm{H}$ & 0.21 & -0.48 & $0.13(\mathrm{H})$ \\
\hline $\mathrm{CF}_{3}$ & $\mathrm{H}$ & 0.29 & -0.44 & $1.05(\mathrm{C})$ \\
\hline $\mathrm{CH}_{3}$ & $\mathrm{H}$ & 0.39 & -0.50 & $-0.79(\mathrm{C})$ \\
\hline $\mathrm{CH}_{3}$ & $\mathrm{CH}_{3}$ & 0.56 & -0.51 & $-0.78(\mathrm{C})$ \\
\hline $\mathrm{CHO}$ & $\mathrm{H}$ & 0.29 & -0.45 & 0.29 (C) \\
\hline $\mathrm{F}$ & $\mathrm{H}$ & 0.73 & -0.50 & $-0.37(F)$ \\
\hline $\mathrm{NH}_{2}$ & $\mathrm{H}$ & 0.50 & -0.58 & $-0.88(\mathrm{~N})$ \\
\hline $\mathrm{CH}=\mathrm{CH}_{2}$ & $\mathrm{H}$ & 0.35 & -0.49 & $-0.33(\mathrm{C})$ \\
\hline $\mathrm{C}_{6} \mathrm{H}_{5}$ & $\mathrm{H}$ & 0.38 & -0.49 & $-0.16(\mathrm{C})$ \\
\hline $\mathrm{COOCH}_{3}$ & $\mathrm{H}$ & 0.31 & -0.44 & $0.69(\mathrm{C})$ \\
\hline
\end{tabular}

\begin{tabular}{|ccc|cccc|}
\hline \multicolumn{2}{|c|}{ Trihydrosilyl Enol Ether } & \multicolumn{2}{c}{ Si } & $\mathrm{O}_{\alpha}$ & $\mathrm{C}_{\alpha}$ & $\mathrm{C}_{\beta}$ \\
$\mathrm{R}_{\mathrm{c}}$ & $\mathrm{R}_{\mathrm{d}}$ & $\mathrm{R}_{\mathrm{e}}$ & & & & \\
\hline \hline $\mathrm{H}$ & $\mathrm{H}$ & $\mathrm{H}$ & 1.26 & -0.86 & 0.14 & -0.55 \\
$\mathrm{CH}_{3}$ & $\mathrm{H}$ & $\mathrm{H}$ & 1.26 & -0.86 & 0.33 & -0.54 \\
$\mathrm{CHO}$ & $\mathrm{H}$ & $\mathrm{H}$ & 1.26 & -0.85 & 0.20 & -0.46 \\
$\mathrm{~F}$ & $\mathrm{H}$ & $\mathrm{H}$ & 1.27 & -0.85 & 0.73 & -0.64 \\
$\mathrm{NH}_{2}$ & $\mathrm{H}$ & $\mathrm{H}$ & 1.28 & -0.87 & 0.50 & -0.62 \\
$\mathrm{OH}$ & $\mathrm{H}$ & $\mathrm{H}$ & 1.27 & -0.88 & 0.64 & -0.64 \\
$\mathrm{SH}$ & $\mathrm{H}$ & $\mathrm{H}$ & 1.26 & -0.85 & 0.15 & -0.54 \\
\hline
\end{tabular}

${ }^{\mathrm{a}}$ Based on MP2(full)/6-31G* wavefunction. 
Table S8. Cartesian Coordinates (Á) of Optimized MP2(full)/6-31G* Geometries of Reactants, Transition States, Intermediate and Products

Reactant $\left(\mathbf{R}_{\mathrm{a}}=\mathrm{R}_{\mathrm{b}}=\mathrm{H}\right)$

Energy: -114.17496 hartree

$\begin{array}{lll}0 & 0.000000 & 0.000000\end{array}$

$\begin{array}{lll}C & 0.0000000 & 0.000000\end{array}$

$\begin{array}{lll}H & 0.934476 & 0.000000\end{array}$

H $\quad-0.934476 \quad 0.000000$

Reactant $\left(\mathrm{R}_{\mathrm{c}}=\mathrm{R}_{\mathrm{d}}=\mathrm{R}_{\mathrm{e}}=\mathrm{H}\right)$

Energy: -443.53532 hartree

$\begin{array}{lll}\text { C } & 0.248719 & 0.000000\end{array}$

H $\quad 0.101059 \quad 0.000000$

H $\quad 1.270442 \quad 0.000000$

$\begin{array}{lll}\text { C } & -0.820986 & 0.000000\end{array}$

H $\quad-1.834029 \quad 0.000000$

$0 \quad-0.826729$

Si $\quad 0.550954$

H 1.377496

H $\quad 1.377496$

H $\quad 0.041611$

0.000000

0.000000

1.207093

$-1.207093$

$\odot .000000$

Transition State $\left(\mathbf{R}_{\mathrm{a}}=\mathrm{R}_{\mathrm{b}}=\mathrm{R}_{\mathrm{c}}=\mathbf{R}_{\mathrm{d}}=\mathbf{R}_{\mathrm{e}}=\mathrm{H}\right)$

Energy: -557.68940 hartree

Imaginary Frequency: $-548 \mathrm{~cm}^{-1}$

$\begin{array}{lr}\text { O } & 0.000000 \\ \text { C } & 0.000000 \\ \text { H } & 0.969098 \\ \text { H } & -0.810524 \\ \text { C } & -0.722977 \\ \text { H } & -0.299314 \\ \text { H } & -1.743669 \\ \text { C } & -0.283693 \\ \text { H } & 0.580159 \\ \text { O } & -0.716000 \\ \text { Si } & -1.597354 \\ \text { H } & -2.632398 \\ \text { H } & -1.047732 \\ \text { H } & -2.432548\end{array}$

0.000000

0.000000

$\odot .000000$

$-0.505750$

1.833016

2.118265

1.469793

2.489316

3.157459

2.215215

0.743657

1.613652

0.011259

$\odot .002224$

Product $\left(\mathrm{R}_{\mathrm{a}}=\mathrm{R}_{\mathrm{b}}=\mathrm{R}_{\mathrm{c}}=\mathrm{R}_{\mathrm{d}}=\mathrm{R}_{\mathrm{e}}=\mathrm{H}\right)$

Energy: -557.75992 hartree

$\begin{array}{lll}0 & 0.386515 & -0.821087\end{array}$

$\begin{array}{lll}\text { C } & 0.351229 & -1.083736\end{array}$

$\mathrm{H} \quad 1.348531-1.444006$

H $\quad-0.365709 \quad-1.880553$

$\begin{array}{lll}\text { C } & 0.020593 & 0.185143\end{array}$

$\begin{array}{lll}\text { H } & 0.123836 & -0.009930\end{array}$

$\begin{array}{lll}H & -1.000473 & 0.526347\end{array}$

$\begin{array}{lll}\text { C } & 0.969286 & 1.287942\end{array}$

H 2.046030

$0 \quad 0.617057$

Si $\quad-1.001242$

H $\quad-1.868387$

H $\quad-0.546939$

H $\quad-1.794735$
$-0.683479$

0.536364

1.124822

1.124822

1.902590

2. 974451

1.540614

1.101425

1.492577

$-0.269040$

$-1.234918$

$-0.984864$

$-0.984864$

$-2.620837$
Reactant $\left(\mathrm{R}_{\mathrm{a}}=\mathrm{CF}_{3} ; \mathrm{R}_{\mathrm{b}}=\mathrm{H}\right)$

Energy: -450.43716 hartree

$\begin{array}{lll}0 & 1.153676 & 0.295194\end{array}$

$\begin{array}{llll}\text { H } & 1.953337 & -0.428703 & 0.078720\end{array}$

$\begin{array}{llll}\text { C } & -0.221066 & -0.044049 & 0.279322\end{array}$

$\begin{array}{llll}F & -1.238938 & 0.402695 & -0.456782\end{array}$

$\begin{array}{llll}F & -0.080553 & 0.746778 & 1.363975\end{array}$

$\begin{array}{llll}F & -0.501794 & -1.292361 & 0.708896\end{array}$

Transition State $\left(\mathbf{R}_{\mathrm{a}}=\mathrm{CF}_{3} ; \mathbf{R}_{\mathrm{b}}=\mathbf{R}_{\mathrm{c}}=\mathbf{R}_{\mathrm{d}}=\mathbf{R}_{\mathrm{e}}=\mathbf{H}\right)$

Energy: -893.96048 hartree

Imaginary Frequency: $-535 \mathrm{~cm}^{-1}$

$\begin{array}{lrrr}\text { O } & 0.457178 & 0.452100 & -0.999984 \\ \mathrm{C} & 0.674334 & 0.431124 & 0.249566 \\ \mathrm{H} & 1.697799 & 0.335290 & 0.644276 \\ \mathrm{C} & 0.154003 & -1.420340 & 0.994138 \\ \mathrm{H} & -0.102843 & -1.359697 & 2.045213 \\ \mathrm{H} & 1.133977 & -1.821386 & 0.761813 \\ \mathrm{C} & -0.864001 & -1.583257 & 0.078738 \\ \mathrm{H} & -1.912986 & -1.487829 & 0.358525 \\ \mathrm{O} & -0.660348 & -1.652260 & -1.200026 \\ \mathrm{Si} & 0.811627 & -1.329344 & -2.143633 \\ \mathrm{H} & 0.777731 & -2.666231 & -2.815019 \\ \mathrm{H} & 0.647513 & -0.372138 & -3.252429 \\ \mathrm{H} & 2.099307 & -1.358592 & -1.405202 \\ \mathrm{C} & -0.200171 & 1.340732 & 1.093196 \\ \mathrm{~F} & -1.508786 & 1.154774 & 0.843076 \\ \mathrm{~F} & 0.094291 & 2.628650 & 0.828034 \\ \mathrm{~F} & 0.007505 & 1.142479 & 2.415550\end{array}$

Product $\left(\mathrm{R}_{\mathrm{a}}=\mathrm{CF}_{3} ; \mathrm{R}_{\mathrm{b}}=\mathrm{R}_{\mathrm{c}}=\mathrm{R}_{\mathrm{d}}=\mathrm{R}_{\mathrm{e}}=\mathrm{H}\right)$

Energy: -894.03117 hartree

$0 \quad 0.257781 \quad-0.660846$

$-0.386815$

$-0.954723$

$-1.609913$

$-2.118176$

0.035656

$-0.663940$

$\odot .737714$

1.009797

0.976831

1.511402

2. 588026

1.320932

1.118951

1.182800

0.751942

$-1.595797$

$-1.275514$

$-2.999027$

$-1.375097$
๑. 328695

1. 276248

$-0.840936$

$-0.999654$

$-0.612840$

$-2.128450$

$-2.377710$

$-2.878589$

1.580862

1. 324938

1. 733182

๑. 334538

$-0.773278$

1. 397602

๑. 433901
2.803129
$-0.382053$

$-0.735005$

$-1.079455$

$-0.658085$

$-2.141681$

$-0.989991$

$-0.011081$

$-1.946247$

$-0.637726$

$-0.942010$

$-1.691314$

๑. 667956

1. 119326

1.722480

1.679820

1. 407609
$-0.925995$

0.457794

๑. 890569

1. 139315

2. 140079

1. 257336

○. 345125

$-0.095274$

๑. 218414

$-1.969794$

$-1.182230$

$-2.974652$

$-2.649392$

๑. 673178

๑. 201349

๑. 049325

1. 989200 
Reactant $\left(\mathrm{R}_{\mathrm{a}}=\mathrm{CH}_{3} ; \mathrm{R}_{\mathrm{b}}=\mathrm{H}\right)$

Energy: -153.35897 hartree

C $\quad 0.183406 \quad 0.095014$

$\mathrm{H} \quad 0.145917 \quad 0.415310$

$0 \quad 1.247596 \quad-0.220319$

C $\quad-1.136632 \quad 0.085702$

$\mathrm{H} \quad-1.576985 \quad 1.087841$

H $\quad-0.996335 \quad-0.241507$

$\mathrm{H} \quad-1.834012$ $-\odot .583391$

Reactant $\left(\mathbf{R}_{\mathrm{a}}=\mathrm{R}_{\mathrm{b}}=\mathrm{CH}_{3}\right)$

Energy: -192.54087 hartree

$\begin{array}{lll}0 & 0.000000 & 0.000000\end{array}$

$\begin{array}{llll}C & 0.000000 & 0.000000 & -0.179360\end{array}$

$\begin{array}{llll}C & 1.282002 & 0.093567 & 0.616372\end{array}$

$\begin{array}{llll}\mathrm{H} & 1.263558 & 0.975522 & 1.264647\end{array}$

H $\quad 1.392040 \quad-0.782031 \quad 1.264194$

$\begin{array}{llll}\mathrm{H} & 2.129710 & 0.155684 & -0.066203\end{array}$

C $\quad-1.282002 \quad-0.093567 \quad 0.616372$

$\begin{array}{llll}\mathrm{H} & -1.263558 & -0.975522 & 1.264647\end{array}$

$\begin{array}{llll}\mathrm{H} & -1.392040 & 0.782031 & 1.264194\end{array}$

$\begin{array}{llll}\mathrm{H} & -2.129710 & -0.155684 & -0.066203\end{array}$

Energy: -596.87086 hartree Imaginary Frequency: $-570 \mathrm{~cm}^{-1}$

$\begin{array}{lrrr}\text { O } & 0.000 \odot \odot \odot & 0.000 \odot \odot \odot & 0.000 \odot \odot \odot \\ \mathrm{C} & 0.000 \odot \odot \odot & 0.000 \odot \odot \odot & 1.287326 \\ \mathrm{H} & 0.978325 & 0.0000 \odot \odot & 1.789215 \\ \mathrm{C} & -0.292701 & -1.949197 & 1.892975 \\ \mathrm{H} & -0.781984 & -2.002612 & 2.859891 \\ \mathrm{H} & 0.772191 & -2.151439 & 1.887909 \\ \mathrm{C} & -1.022502 & -2.296189 & 0.773364 \\ \mathrm{H} & -2.102790 & -2.456219 & 0.819238 \\ \mathrm{O} & -0.533523 & -2.260332 & -0.422124 \\ \mathrm{Si} & 0.971742 & -1.397522 & -0.977798 \\ \mathrm{H} & 1.404156 & -2.661714 & -1.669220 \\ \mathrm{H} & 0.873522 & -0.468139 & -2.128507 \\ \mathrm{H} & 2.092488 & -1.222158 & -0.004229 \\ \mathrm{C} & -1.084061 & 0.786139 & 1.972234 \\ \mathrm{H} & -1.106055 & 0.592200 & 3.046621 \\ \mathrm{H} & -0.885314 & 1.851896 & 1.815176 \\ \mathrm{H} & -2.054800 & 0.553973 & 1.531855\end{array}$

Product $\left(\mathrm{R}_{\mathrm{a}}=\mathrm{CH}_{3} ; \mathrm{R}_{\mathrm{b}}=\mathrm{R}_{\mathrm{c}}=\mathrm{R}_{\mathrm{d}}=\mathrm{R}_{\mathrm{e}}=\mathrm{H}\right)$

Energy: -596.93643 hartree

$-0.396808$

$-0.496317$
0.430589

0.460816

0.560026

1.492782

0.678613

$\odot .560939$

$-0.436115$

$-0.553241$

0.097952

$-0.235447$

$-1.406757$

$-1.728726$

$-2.379264$

$-0.542547$

$\odot .352392$

$-1.548079$

$-1.919537$

$-0.242997$

$-0.218448$

2. 078082

2. 233056

2. 812197

2. 249258

-0.693837
0.735101
1.099325
1.344396
2.412699
1.247064
0.647625
0.690359
0.059993
-1.522636
-1.202824
-2.957763
-1.182106
1.075677
2.158463
0.619987
0.685649

0.650721
-1.022178
Transition State $\left(\mathrm{R}_{\mathrm{a}}=\mathrm{R}_{\mathrm{b}}=\mathrm{CH}_{3} ; \mathrm{R}_{\mathrm{c}}=\mathrm{R}_{\mathrm{d}}=\mathrm{R}_{\mathrm{e}}=\mathrm{H}\right)$

Energy: -636.04852 hartree Imaginary Frequency: $-577 \mathrm{~cm}^{-1}$

$\begin{array}{lrrr}\text { O } & -0.929762 & -0.033819 & -0.528987 \\ \text { C } & -0.882526 & -0.089150 & 0.760078 \\ \text { C } & 1.118529 & -0.082408 & 1.231936 \\ \text { H } & 1.268523 & -0.440312 & 2.245070 \\ \text { H } & 1.202470 & 0.987850 & 1.085866 \\ \text { C } & 1.467742 & -0.911723 & 0.187338 \\ \text { H } & 1.710506 & -1.965111 & 0.348600 \\ \text { O } & 1.354203 & -0.570642 & -1.055544 \\ \text { Si } & 0.397551 & 0.792972 & -1.762793 \\ \text { H } & 1.555160 & 1.080636 & -2.679691 \\ \text { H } & -0.676883 & 0.551623 & -2.755096 \\ \text { H } & 0.353449 & 2.047805 & -0.963414 \\ \text { C } & -1.384425 & -1.384564 & 1.363244 \\ \text { H } & -1.117105 & -1.480068 & 2.418286 \\ \text { H } & -2.477503 & -1.390356 & 1.281712 \\ \text { H } & -0.999435 & -2.235629 & 0.800036 \\ \text { C } & -1.258985 & 1.162514 & 1.531177 \\ \text { H } & -2.349947 & 1.256515 & 1.475430 \\ \text { H } & -0.970832 & 1.103410 & 2.583215 \\ \text { H } & -0.821651 & 2.049701 & 1.072685\end{array}$

Product $\left(\mathrm{R}_{\mathrm{a}}=\mathrm{R}_{\mathrm{b}}=\mathrm{CH}_{3} ; \mathrm{R}_{\mathrm{c}}=\mathrm{R}_{\mathrm{d}}=\mathrm{R}_{\mathrm{e}}=\mathrm{H}\right)$

Energy: -636.11443 hartree

$0-0.595695 \quad 0.008529$

$\begin{array}{llll}\text { C } & -0.678414 & -0.088782 & 0.663286\end{array}$

$\begin{array}{llll}\text { C } & 0.745753 & -0.271351 & 1.225915\end{array}$

$\begin{array}{llll}\mathrm{H} & 0.682330 & -0.513119 & 2.293868\end{array}$

$\begin{array}{llll}\mathrm{H} & 1.308481 & 0.659527 & 1.110253\end{array}$

$\begin{array}{llll}\text { C } & 1.491239 & -1.350832 & 0.489141\end{array}$

$\begin{array}{llll}\mathrm{H} & 1.292359 & -2.396267 & 0.798139\end{array}$

$\begin{array}{llll}0 & 2.276084 & -1.118290 & -0.420976\end{array}$

Si $\quad 0.020599 \quad 1.270539 \quad-1.671207$

$\begin{array}{llll}\mathrm{H} & 1.159790 & 1.953218 & -1.006760\end{array}$

$\begin{array}{llll}H & 0.449627 & 0.681977 & -2.957361\end{array}$

$\begin{array}{llll}\mathrm{H} & -1.026118 & 2.296192 & -1.930139\end{array}$

$\begin{array}{llll}\text { C } & -1.546412 & -1.310444 & 0.930269\end{array}$

$\begin{array}{llll}\text { H } & -1.636829 & -1.499776 & 2.004042\end{array}$

$\begin{array}{llll}\mathrm{H} & -2.543520 & -1.140750 & 0.516740\end{array}$

$\begin{array}{llll}\text { H } & -1.128179 & -2.194641 & 0.444718\end{array}$

$\begin{array}{llll}\text { C } & -1.319183 & 1.159371 & 1.256263\end{array}$

$\begin{array}{llll}\mathrm{H} & -2.306387 & 1.313553 & 0.812119\end{array}$

$\begin{array}{llll}H & -1.434879 & 1.057398 & 2.339453\end{array}$

$\begin{array}{llll}H & -0.706066 & 2.045459 & 1.064403\end{array}$ 
Reactant ( $\left.\mathrm{R}_{\mathrm{a}}=\mathrm{CHO} ; \mathbf{R}_{\mathrm{b}}=\mathrm{H}\right)$ Energy: -227.20373 hartree $\begin{array}{lll}\text { C } & 0.668072 & 0.00000 \odot\end{array}$ $\begin{array}{lll}\mathrm{H} & 0.615739 & 0.000000\end{array}$

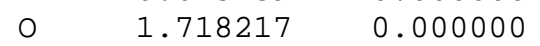

$\begin{array}{lll}C & -0.668072 & 0.00000 \odot\end{array}$

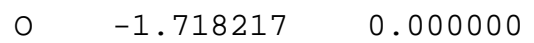

H $\quad-0.615739$

$\odot .000000$
0.354777

1.459164

$-0.271972$

$-0.354777$

๑. 271972

$-1.459164$

Transition State $\left(\mathbf{R}_{\mathrm{a}}=\mathrm{CHO} ; \mathbf{R}_{\mathrm{b}}=\mathbf{R}_{\mathrm{c}}=\mathbf{R}_{\mathrm{d}}=\mathbf{R}_{\mathrm{e}}=\mathrm{H}\right)$ Energy: -670.72298 hartree Imaginary Frequency: $-547 \mathrm{~cm}^{-1}$

$\begin{array}{lrrr}\text { O } & 0.227185 & 0.859881 & -0.722605 \\ \mathrm{C} & 0.338908 & 0.856272 & 0.556016 \\ \mathrm{H} & 1.333076 & 0.820289 & 1.023700 \\ \mathrm{C} & -0.121020 & -1.024635 & 1.239931 \\ \mathrm{H} & -0.530244 & -0.990758 & 2.244412 \\ \mathrm{H} & 0.916309 & -1.330306 & 1.159247 \\ \mathrm{C} & -0.971122 & -1.305484 & 0.184143 \\ \mathrm{H} & -2.055256 & -1.350364 & \odot .313571 \\ \mathrm{O} & -0.572815 & -1.329298 & -1.043048 \\ \mathrm{Si} & 0.968212 & -0.695307 & -1.765333 \\ \mathrm{H} & 1.187078 & -2.016272 & -2.441858 \\ \mathrm{H} & 0.856938 & 0.227127 & -2.916116 \\ \mathrm{H} & 2.163291 & -0.611260 & -0.879227 \\ \mathrm{C} & -0.697269 & 1.629684 & 1.319407 \\ \mathrm{H} & -1.614692 & 1.843590 & 0.735858 \\ \mathrm{O} & -0.542927 & 1.995320 & 2.475415\end{array}$

Product $\left(\mathrm{R}_{\mathrm{a}}=\mathrm{CHO} ; \mathbf{R}_{\mathrm{b}}=\mathrm{R}_{\mathrm{c}}=\mathrm{R}_{\mathrm{d}}=\mathrm{R}_{\mathrm{e}}=\mathrm{H}\right)$

Energy: -670.78909 hartree

$\begin{array}{lrrr}\text { O } & 0.500448 & 0.396912 & -0.928301 \\ \mathrm{C} & 0.546655 & 0.487437 & 0.492120 \\ \mathrm{H} & 1.590482 & 0.452564 & 0.849371 \\ \mathrm{C} & -0.258227 & -0.620058 & 1.164033 \\ \mathrm{H} & -0.376773 & -0.359591 & 2.222995 \\ \mathrm{H} & \odot .244529 & -1.587779 & 1.088101 \\ \mathrm{C} & -1.615733 & -0.738496 & 0.518175 \\ \mathrm{H} & -2.225737 & 0.186254 & 0.510262 \\ \mathrm{O} & -2.042788 & -1.773243 & 0.028213 \\ \mathrm{Si} & 1.408208 & -0.733691 & -1.777820 \\ \mathrm{H} & 0.913648 & -2.116464 & -1.578050 \\ \mathrm{H} & 1.296734 & -0.346613 & -3.198549 \\ \mathrm{H} & 2.820917 & -0.660524 & -1.316896 \\ \mathrm{C} & 0.012251 & 1.867643 & 0.841084 \\ \mathrm{H} & 0.128496 & 2.618910 & 0.036959 \\ \mathrm{O} & -0.484770 & 2.139552 & 1.922939\end{array}$

Reactant $\left(\mathbf{R}_{\mathrm{a}}=\mathrm{F} ; \mathbf{R}_{\mathrm{b}}=\mathrm{H}\right)$

Energy: -213.22926 hartree

$\begin{array}{lll}0 & 0.370409 & 0.000000\end{array}$

$\begin{array}{llll}C & 0.389590 & 0.000000 & 0.085956\end{array}$

$\begin{array}{llll}\text { H } & 1.266624 & 0.000 \odot \odot \odot ~ & 0.740021\end{array}$

$\begin{array}{llll} & -0.729715 & 0.000 \odot \odot \odot ~ & 0.844928\end{array}$

Transition state $\left(\mathbf{R}_{\mathrm{a}}=\mathrm{F} ; \mathbf{R}_{\mathrm{b}}=\mathbf{R}_{\mathrm{c}}=\mathbf{R}_{\mathrm{d}}=\mathbf{R}_{\mathrm{e}}=\mathrm{H}\right)$

Energy: -656.73096 hartree

Imaginary Frequency: $-583 \mathrm{~cm}^{-1}$

$\begin{array}{lrrr}\text { O } & 0.749675 & 0.894849 & -0.412028 \\ \mathrm{C} & 0.815450 & 0.865290 & 0.842647 \\ \mathrm{H} & 1.784004 & 0.849734 & 1.358975 \\ \mathrm{C} & 0.218880 & -0.817629 & 1.433665 \\ \mathrm{H} & 0.217746 & -0.837894 & 2.518507 \\ \mathrm{H} & 0.997245 & -1.402586 & 0.952840 \\ \mathrm{C} & -1.012850 & -0.721270 & 0.803511 \\ \mathrm{H} & -1.913546 & -0.442033 & 1.352202 \\ \mathrm{O} & -1.151672 & -0.724740 & -0.478419 \\ \mathrm{Si} & \odot .090187 & -0.628340 & -1.764161 \\ \mathrm{H} & -0.619203 & -1.669059 & -2.572588 \\ \mathrm{H} & 0.085090 & 0.546982 & -2.653831 \\ \mathrm{H} & 1.378707 & -1.274505 & -1.429111 \\ \mathrm{~F} & -0.011730 & 1.745211 & 1.535099\end{array}$

Product $\left(\mathbf{R}_{\mathrm{a}}=\mathrm{F} ; \mathbf{R}_{\mathrm{b}}=\mathbf{R}_{\mathrm{c}}=\mathbf{R}_{\mathrm{d}}=\mathbf{R}_{\mathrm{e}}=\mathrm{H}\right)$

Energy: -656.79785 hartree$$
0
$$

H

C

$\mathrm{H}$

$H$
$C$
$H$

H $\quad-1.898570$

$\begin{array}{lll}-1.6747958 & -0.199937\end{array}$

Si $\quad 1.584362-0.144415$

H $\quad 2.922471 \quad-0.312129$

$\mathrm{H} \quad 1.393905 \quad-1.097534$

$\mathrm{H} \quad 1.428646 \quad 1.242983$

F $\quad 0.834727 \quad 1.203656$
$-0.600250$

0.726312

1. 192672

1. 451714

2.473237

1. 504488

0.753928

0.355576

0.650809

$-1.739187$

$-1.118331$

$-2.851453$

- 2.227941

$\odot .780463$ 
Reactant $\left(\mathbf{R}_{\mathrm{a}}=\mathrm{NH}_{2} ; \mathbf{R}_{\mathrm{b}}=\mathrm{H}\right)$

Energy: -169.40539 hartree

C $\quad 0.385981 \quad-0.169971$

$0 \quad 0.368027 \quad 0.177015$

$\mathrm{H} \quad 1.253386 \quad-0.672553$

$\mathrm{N} \quad-0.629113 \quad 0.033270$

$\mathrm{H} \quad-1.498532 \quad 0.417257$

H $\quad-0.611164 \quad-0.373883$

Transition state $\left(\mathbf{R}_{\mathrm{a}}=\mathrm{NH}_{2} ; \mathbf{R}_{\mathrm{b}}=\mathbf{R}_{\mathrm{c}}=\mathbf{R}_{\mathrm{d}}=\mathbf{R}_{\mathrm{e}}=\mathrm{H}\right)$ Energy: -612.89842 hartree Imaginary Frequency: $-636 \mathrm{~cm}^{-1}$

$\begin{array}{lrr}\text { O } & 0.144541 & 0.989162 \\ \mathrm{C} & 0.239473 & 0.996648 \\ \mathrm{H} & 1.248609 & 1.037398 \\ \mathrm{C} & -0.055430 & -0.813715 \\ \mathrm{H} & -0.378075 & -0.845273 \\ \mathrm{H} & 0.967862 & -1.141337 \\ \mathrm{C} & -0.975728 & -1.216553 \\ \mathrm{H} & -2.034408 & -1.373966 \\ \mathrm{O} & -0.679172 & -1.228739 \\ \mathrm{Si} & 0.850154 & -0.371833 \\ \mathrm{H} & 1.008495 & -1.659535 \\ \mathrm{H} & 0.744053 & 0.582701 \\ \mathrm{H} & 2.121752 & -0.385600 \\ \mathrm{~N} & -0.621370 & 1.908228 \\ \mathrm{H} & -1.546719 & 1.916131 \\ \mathrm{H} & -0.656972 & 1.835886\end{array}$

Product $\left(\mathbf{R}_{\mathrm{a}}=\mathrm{NH}_{2} ; \mathbf{R}_{\mathrm{b}}=\mathbf{R}_{\mathrm{c}}=\mathrm{R}_{\mathrm{d}}=\mathrm{R}_{\mathrm{e}}=\mathrm{H}\right)$

Energy: -612.95622 hartree

$\begin{array}{lrrr}\mathrm{O} & 0.484808 & 0.618159 & -0.644316 \\ \mathrm{C} & 0.511680 & 0.580961 & 0.777821 \\ \mathrm{H} & 1.548821 & 0.387247 & 1.110968 \\ \mathrm{C} & -0.408282 & -0.523479 & 1.285459 \\ \mathrm{H} & -0.551060 & -0.410768 & 2.368948 \\ \mathrm{H} & 0.017612 & -1.514343 & 1.102464 \\ \mathrm{C} & -1.747027 & -0.449061 & 0.590007 \\ \mathrm{H} & -2.225823 & 0.544396 & 0.581577 \\ \mathrm{O} & -2.277298 & -1.419174 & 0.065182 \\ \mathrm{Si} & 1.407701 & -0.415182 & -1.586645 \\ \mathrm{H} & 0.980983 & -1.830951 & -1.461424 \\ \mathrm{H} & 1.232357 & 0.049379 & -2.977726 \\ \mathrm{H} & 2.837433 & -0.321767 & -1.182929 \\ \mathrm{~N} & -0.002380 & 1.869246 & 1.205093 \\ \mathrm{H} & 0.138128 & 1.980410 & 2.208901 \\ \mathrm{H} & 0.532079 & 2.601815 & 0.739953\end{array}$

$-0.443643$

$\odot .866924$

1.297609

1. 443190

2.480724

1.283803

0.471816

$\odot .705399$

$-0.770716$

$-1.507292$

$-2.272107$

$-2.640387$

$-0.716273$

1.488059

1.071119

2.499084

\section{(1)}

$-\odot .015978$

Reactant $\left(\mathrm{R}_{\mathrm{a}}=\mathrm{C}_{2} \mathrm{H}_{3} ; \mathbf{R}_{\mathrm{b}}=\mathrm{H}\right)$

Energy: -191.32858 hartree

$\begin{array}{lrrr}0 & 0.671522 & 0.00000 \odot & -1.674262 \\ \mathrm{C} & 0.609499 & 0.00000 \odot & -0.449903 \\ \mathrm{H} & 1.531045 & 0.000000 & 0.169706 \\ \mathrm{C} & -0.650597 & 0.00000 \odot & 0.309026 \\ \mathrm{H} & -1.572724 & 0.00000 \odot & -0.265507 \\ \mathrm{C} & -0.638628 & 0.00000 \odot & 1.649548 \\ \mathrm{H} & -1.550236 & 0.000000 & 2.236666 \\ \mathrm{H} & 0.298096 & 0.00000 \odot & 2.201204\end{array}$

Transition state $\left(\mathbf{R}_{\mathrm{a}}=\mathrm{C}_{2} \mathrm{H}_{3} ; \mathbf{R}_{\mathrm{b}}=\mathbf{R}_{\mathrm{c}}=\mathbf{R}_{\mathrm{d}}=\mathbf{R}_{\mathrm{e}}=\mathrm{H}\right)$

Energy: -634.83879 hartree

Imaginary Frequency: $-577 \mathrm{~cm}^{-1}$

$\begin{array}{lrrr}\mathrm{O} & 0.143583 & 0.802243 & -0.719301 \\ \mathrm{C} & 0.207616 & 0.802438 & 0.574431 \\ \mathrm{H} & 1.207376 & 0.801879 & 1.035179 \\ \mathrm{C} & -0.110570 & -1.110618 & 1.186495 \\ \mathrm{H} & -0.547632 & -1.127508 & 2.179137 \\ \mathrm{H} & 0.944539 & -1.354814 & 1.128129 \\ \mathrm{C} & -0.908633 & -1.457202 & 0.109207 \\ \mathrm{H} & -1.988637 & -1.593302 & 0.213247 \\ \mathrm{O} & -0.476850 & -1.438929 & -1.104963 \\ \mathrm{Si} & 1.027083 & -0.591095 & -1.735495 \\ \mathrm{H} & 1.376838 & -1.868615 & -2.449368 \\ \mathrm{H} & 0.909561 & 0.341458 & -2.882871 \\ \mathrm{H} & 2.205426 & -0.461372 & -0.823121 \\ \mathrm{C} & -0.813838 & 1.585492 & 1.295169 \\ \mathrm{H} & -1.778272 & 1.674855 & 0.800223 \\ \mathrm{C} & -0.563630 & 2.189907 & 2.463625 \\ \mathrm{H} & -1.315177 & 2.784063 & 2.971402 \\ \mathrm{H} & 0.407289 & 2.112065 & 2.945516\end{array}$

Product $\left(\mathrm{R}_{\mathrm{a}}=\mathrm{C}_{2} \mathrm{H}_{3} ; \quad \mathbf{R}_{\mathrm{b}}=\mathrm{R}_{\mathrm{c}}=\mathrm{R}_{\mathrm{d}}=\mathrm{R}_{\mathrm{e}}=\mathrm{H}\right)$

Energy: -634.90206 hartree

$\begin{array}{lrrr}\mathrm{O} & 0.385892 & -0.480118 & -0.743912 \\ \mathrm{C} & 0.292529 & -0.088801 & 0.636235 \\ \mathrm{H} & 1.189366 & -0.429610 & 1.176215 \\ \mathrm{C} & -0.921005 & -0.828375 & 1.190974 \\ \mathrm{H} & -1.128437 & -0.474111 & 2.209940 \\ \mathrm{H} & -0.721510 & -1.902738 & 1.234164 \\ \mathrm{C} & -2.151415 & -0.629812 & 0.340939 \\ \mathrm{H} & -2.346695 & 0.402711 & -0.008785 \\ \mathrm{O} & -2.926123 & -1.531761 & 0.059422 \\ \mathrm{Si} & 1.702898 & -0.027859 & -1.679281 \\ \mathrm{H} & 2.960058 & -0.228699 & -0.908951 \\ \mathrm{H} & 1.673209 & -0.911264 & -2.864632 \\ \mathrm{H} & 1.638317 & 1.388845 & -2.118087 \\ \mathrm{C} & 0.203877 & 1.402993 & 0.759154 \\ \mathrm{H} & -0.592603 & 1.897676 & 0.206225 \\ \mathrm{C} & 1.066532 & 2.124698 & 1.484091 \\ \mathrm{H} & 0.986281 & 3.203694 & 1.561738 \\ \mathrm{H} & 1.880193 & 1.654346 & 2.029678\end{array}$


Reactant $\left(\mathrm{R}_{\mathrm{a}}=\mathrm{C}_{6} \mathrm{H}_{5} ; \mathrm{R}_{\mathrm{b}}=\mathrm{H}\right)$

Energy: -344.52370 hartree

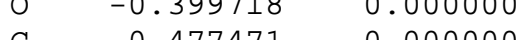

$\begin{array}{lll}\mathrm{C} & 0.477471 & 0.000000 \\ \mathrm{H} & 1.549083 & 0.000001\end{array}$

H $\quad 1.549083$

C $\quad 0.219617$

C $\quad-1.096625$

C -1.331413

C $\quad-0.257832$

C $\quad 1.054132$

C $\quad 1.294071$

H $\quad-1.911976$

$\mathrm{H} \quad-2.351114$

H $\quad-0.445694$

H 1.886486

H 2.314438

0.000001

$\odot .000000$

0.000000

0.000000

0.000000

$\odot .000000$

$\odot .000000$

$\odot .0 \odot \odot \odot \odot \odot$

0.000000

0.000000

0.000000

0.000000

$-2.841773$

$-1.984919$

$-2.274961$

$-0.529583$

$-0.053568$

1. 316906

2. 212920

1.737456

$\odot .364750$

$-0.772167$

1. 693345

3. 283740

2. 436329

$-0.015863$

Transition State $\left(\mathbf{R}_{\mathrm{a}}=\mathrm{C}_{6} \mathbf{H}_{5} ; \mathbf{R}_{\mathrm{b}}=\mathbf{R}_{\mathrm{c}}=\mathbf{R}_{\mathrm{d}}=\mathbf{R}_{\mathrm{e}}=\mathrm{H}\right)$ Energy: -788.03618 hartree Imaginary Frequency: $-580 \mathrm{~cm}^{-1}$

0

0.889510

0.886602

0.100120

0.083266

0.042466

1.862601

0.507638

$-1.829903$

0.056485

1.551595

$-0.294072$

$-1.842301$

$-2.119047$

$-2.114610$

$-2.185603$

$-2.098341$

$\odot .152896$

$-1.344999$

$-2.633201$

$-0.395583$

$-1.284939$

$\odot .880598$

1.299466

2. 049671

2. 385227

1.972712

1.222683

1. 043406

2. 378941

2. 971942

2. 240252

0.904748

$-1.244552$

○. 049156

$\odot .557215$

0.627957

1. 614447

0.576688

$-0.463726$

$-0.374885$

$-1.673852$

$-2.254802$

$-2.972638$

$-3.392969$

$-1.311444$

0.749362

0.062705

0.724437

2. 070485

2. 753649

2.094523

$-\odot .988027$

0.188968

2. 583197

3. 798265

2.624355
Product $\left(\mathrm{R}_{\mathrm{a}}=\mathrm{C}_{6} \mathrm{H}_{5} ; \mathrm{R}_{\mathrm{b}}=\mathrm{R}_{\mathrm{c}}=\mathrm{R}_{\mathrm{d}}=\mathrm{R}_{\mathrm{e}}=\mathrm{H}\right)$

Energy: -788.09719 hartree

$\begin{array}{lll}0 & 0.000000 & 0.000000\end{array}$

$\begin{array}{lll}\text { C } & 0.0000000 & 0.000000\end{array}$

$\begin{array}{lll}\mathrm{H} & 1.035218 & 0.000000\end{array}$

$\begin{array}{lll}\text { C } & -0.703244 & -1.272108\end{array}$

H $\quad-0.834328-1.218150$

$\mathrm{H} \quad-0.115198-2.164645$

$\begin{array}{lll}\mathrm{C} & -2.049473 & -1.417679 \\ \mathrm{H} & -2.740682 & -0.562210\end{array}$

$-2.383030 \quad-2.407995$

$1.161692-0.818631$

$1.064918-2.291637$

$0.925048-0.443992$

2.518248

$-0.687981$

$-\odot .392311$

1. 251684

2. 060135

3. 192803

$-2.085394$

$-1.988046$

$-1.232039$

3. 526973

2. 725339

1.592920

1. 803174

$-1.501989$

3. 817126

$-2.667263$

$-2.490885$

4. 411463

$-1.141497$

2.983673

$\odot .979730$

0.000000

1.428888

1.806445

1. 920380

3. 008890

1.685588

1. 258164

1.378418

0.622179

$-0.887180$

$-0.729097$

$-2.296777$

$-0.449712$

1. 924678

1. 068695

1.565151

2. 915358

3. 771787

3. 278134

๑. 016284

0.891522

3. 297704

4. 823926

3. 950126 
Reactant $\left(\mathrm{R}_{\mathrm{a}}=\mathrm{COOCH}_{3} ; \mathbf{R}_{\mathrm{b}}=\mathrm{H}\right)$ Energy: - 341.43472 hartree $\begin{array}{lll}\text { C } & 0.368294 & 0.000000\end{array}$ $\mathrm{H} \quad 1.366708 \quad 0.000000$

$0 \quad-0.673522 \quad 0.00000 \odot$

C $\quad 0.439114 \quad 0.00000 \odot$

$0 \quad 1.509586 \quad 0.000000$

$\begin{array}{lll}0 & -0.771983 & 0.000000\end{array}$

$\begin{array}{lll}\text { C } & -0.744967 & 0.000000\end{array}$

$\mathrm{H} \quad-0.232186 \quad 0.890202$

H $\quad-0.232186 \quad-0.890202$

H $\quad-1.789631 \quad 0.0000 \odot \odot$

Transition state

$\left(\mathbf{R}_{\mathrm{a}}=\mathrm{COOCH}_{3} ; \mathbf{R}_{\mathrm{b}}=\mathbf{R}_{\mathrm{c}}=\mathrm{R}_{\mathrm{d}}=\mathrm{R}_{\mathrm{e}}=\mathrm{H}\right)$

Energy: -784.95482 hartree Imaginary Frequency: $-547 \mathrm{~cm}^{-1}$

$\begin{array}{lr}\mathrm{O} & 0.708481 \\ \mathrm{C} & 0.761115 \\ \mathrm{H} & 1.731383 \\ \mathrm{C} & 0.377245 \\ \mathrm{H} & -0.044659 \\ \mathrm{H} & 1.423081 \\ \mathrm{C} & -0.454838 \\ \mathrm{H} & -1.539599 \\ \mathrm{O} & -0.037237 \\ \mathrm{Si} & 1.504373 \\ \mathrm{H} & 1.766765 \\ \mathrm{H} & 1.406452 \\ \mathrm{H} & 2.676655 \\ \mathrm{C} & -0.308150 \\ \mathrm{O} & -0.134188 \\ \mathrm{O} & -1.455862 \\ \mathrm{C} & -2.511175 \\ \mathrm{H} & -3.355958 \\ \mathrm{H} & -2.754343 \\ \mathrm{H} & -2.205735\end{array}$

0.499447

0.468550

0.470568

$-1.450550$

$-1.452946$

$-1.726119$

$-1.709681$

$-1.759424$

$-1.698600$

$-1.031116$

$-2.330070$

$-\odot .080552$

$-0.945076$

1.177921

1.592633

1. 269994

1.949756

1.951074

1.416736

2.967675

$-0.904592$

0.372161

$\odot .887125$

1.003330

2. 003231

$\odot .927064$

$-0.069682$

0.040552

$-1.291972$

$-1.963099$

$-2.667585$

$-3.091488$

$-1.047515$

1.160377

2.295139

0.468843

1.179606

0.494951

2.099917

1. 423034

Product $\left(\mathbf{R}_{\mathrm{a}}=\mathrm{COOCH}_{3} ; \mathbf{R}_{\mathrm{b}}=\mathrm{R}_{\mathrm{c}}=\mathrm{R}_{\mathrm{d}}=\mathrm{R}_{\mathrm{e}}=\mathrm{H}\right)$

Energy: -785.02300 hartree

0

1. 246213

0.118942

$-0.049527$

$\odot .356137$

$-0.240936$

$-1.180938$

0.545014

$-0.409019$

$-0.931500$

$-0.024870$

2.257030

2. 180264

3. 622811

1.946082

$-1.052715$

$-2.219302$

$-0.622652$

$-1.661684$

$-1.146392$

$-2.167235$

$-2.385296$

0.803391

1.813290

$\odot .868183$

1.419509

1.382873

$-0.514011$

$-1.250885$

$-0.819348$

$-\odot .087070$

1.218955

$-0.295720$

$-1.192258$

๑. 302669

$\odot .290622$

$-0.081913$

$-0.556714$

$-0.840219$

$-1.413325$

0.236985
Reactant $\left(\mathbf{R}_{\mathrm{c}}=\mathrm{CH}_{3} ; \quad \mathbf{R}_{\mathrm{d}}=\mathrm{R}_{\mathrm{e}}=\mathrm{H}\right)$ Energy: -482.71499 hartree

$\begin{array}{lll}\text { C } & 0.719968 & 0.111640\end{array}$

$\mathrm{H} \quad 0.659303 \quad 0.165631$

H $\quad 1.711252 \quad 0.129032$

C $\quad-0.403945 \quad 0.028125$

$0 \quad-0.441015 \quad-0.045443$

$\begin{array}{lll}\text { Si } & 0.900182 & -0.044476\end{array}$

$\mathrm{H} \quad 1.687275 \quad 1.205016$

$\mathrm{H} \quad 1.784457-1.204126$

H $\quad 0.342584 \quad-0.138562$

C $\quad-1.787528$

0.001465

$\odot .841440$

$-2.367345$

$-0.918954$

$-2.295889$

๑. 059344

1.571981

2.650884

1.134225

0.847673

$-0.529585$

$-1.542799$

$-1.392392$

$-1.265975$

$-2.907529$

1.411333

1. 018821

1.110986

2.500916

Transition State $\left(\mathbf{R}_{\mathrm{c}}=\mathrm{CH}_{3} ; \mathbf{R}_{\mathrm{a}}=\mathbf{R}_{\mathrm{b}}=\mathbf{R}_{\mathrm{d}}=\mathbf{R}_{\mathrm{e}}=\mathrm{H}\right)$

Energy: -596.87356 hartree Imaginary Frequency: $-527 \mathrm{~cm}^{-1}$

\begin{tabular}{|c|c|c|c|}
\hline 0 & 0.601432 & -1.369260 & $-0.28469 \odot$ \\
\hline C & ๑. 461811 & -1.408603 & 0.982816 \\
\hline $\mathrm{H}$ & 1.365571 & -1.383005 & 1.600130 \\
\hline $\mathrm{H}$ & $-\odot .389618$ & -1.947036 & 1.417854 \\
\hline C & $-\odot .382859$ & 0.396305 & 1.61790 \\
\hline $\mathrm{H}$ & $-\odot .048619$ & $\odot .633949$ & 2.62206 \\
\hline $\mathrm{H}$ & -1.398826 & 0.031215 & 1.52364 \\
\hline C & 0.167075 & 1.077834 & $\odot .55277$ \\
\hline C & 1.392473 & 1.925881 & $\odot .64977$ \\
\hline 0 & -0.222191 & 0.835055 & $-\odot .669300$ \\
\hline Si & $-\odot .959841$ & -0.632250 & -1.37279 \\
\hline $\mathrm{H}$ & -1.981362 & 0.193218 & -2.10244 \\
\hline $\mathrm{H}$ & -0.253505 & -1.291115 & -2.495186 \\
\hline $\mathrm{H}$ & -1.829983 & -1.460819 & -0.48843 \\
\hline $\mathrm{H}$ & 1.621566 & 2.195757 & 1.68154 \\
\hline $\mathrm{H}$ & 2.232266 & 1.371739 & 0.21608 \\
\hline & 1.255353 & 2.832733 & 0.05608 \\
\hline
\end{tabular}

Product $\left(\mathbf{R}_{\mathrm{c}}=\mathrm{CH}_{3} ; \mathbf{R}_{\mathrm{a}}=\mathrm{R}_{\mathrm{b}}=\mathrm{R}_{\mathrm{d}}=\mathrm{R}_{\mathrm{e}}=\mathrm{H}\right)$

Energy: -596.94312 hartree

$0.395132-1.033603$

$\begin{array}{lll}\text { C } & 0.255226 & -1.099977\end{array}$

$\begin{array}{lll}\mathrm{C} & 0.255226 & -1.099977 \\ \mathrm{H} & 1.265433 & -1.207844\end{array}$

$\begin{array}{rrr}\mathrm{H} & 1.265433 & -1.207844 \\ \mathrm{H} & -0.315849 & -1.989637\end{array}$

$\begin{array}{llr}\mathrm{C} & -0.408212 & 0.161342\end{array}$

$\mathrm{H} \quad-0.325644 \quad 0.174086$

$\begin{array}{lll}\mathrm{H} & -0.325644 & 0.174086 \\ \mathrm{H} & -1.470373 & 0.195995\end{array}$

1. 413731

2. 281257

$-0.444067$

$-1.383150$

$-0.606995$

0.813253

1. 217521

1.111629

1. 350309

2.445904

1. 092741

๑. 783206

0.244582

$-1.637382$

$-0.378940$

$-1.404958$

$-1.567116$

$-2.997903$

$-0.299523$

$-2.713502$

$-1.448443$

1.532120

$-1.291922$

2. 081811

1. 275271

$\odot .897861$

2. 201351

○. 838200

1. 903591

2.033274

2. 550904

0.191672

$\odot .648767$ 
Reactant $\left(\mathbf{R}_{\mathrm{c}}=\mathrm{CHO} ; \mathbf{R}_{\mathrm{d}}=\mathbf{R}_{\mathrm{e}}=\mathrm{H}\right)$ Energy: -556.56883 hartree $\begin{array}{lll}\text { C } & 0.885411 & -0.140109\end{array}$ $\begin{array}{lll}\mathrm{H} & 0.717411 & -0.382620\end{array}$

H $\quad 1.896903 \quad 0.078705$

C $\quad-0.180102-0.129969$

$0 \quad-0.203494 \quad 0.146410$

Si $\quad 1.154691$

H $\quad 0.636012$

H $\quad 1.790837$

H $\quad 2.142797$

C $\quad-1.545921$

$\mathrm{H} \quad-2.334919$

$0 \quad-1.792887$

0.542564

0.749552

1.777858

$-0.563051$

$-0.440616$

$-0.397748$

$-0.720713$
1.308835

2.350835

0.987864

$\odot .492972$

$-0.849299$

$-1.769189$

$-3.134917$

$-1.250520$

$-1.738971$

๑. 999191

๑. 225384

2.164671

Transition State $\left(\mathbf{R}_{\mathrm{c}}=\mathrm{CHO} ; \mathbf{R}_{\mathrm{a}}=\mathbf{R}_{\mathrm{b}}=\mathbf{R}_{\mathrm{d}}=\mathbf{R}_{\mathrm{e}}=\mathrm{H}\right)$ Energy: -670.72240 hartree Imaginary Frequency: $-567 \mathrm{~cm}^{-1}$

$\begin{array}{lrrr}\mathrm{O} & 0.502053 & 1.522225 & -0.480587 \\ \mathrm{C} & 0.585577 & 1.511460 & 0.800334 \\ \mathrm{H} & 1.576727 & 1.543972 & 1.269186 \\ \mathrm{C} & 0.313750 & -0.396891 & 1.455850 \\ \mathrm{H} & -0.160982 & -0.407632 & 2.431822 \\ \mathrm{H} & 1.370267 & -0.634871 & 1.419244 \\ \mathrm{C} & -0.472644 & -0.740639 & 0.372534 \\ \mathrm{O} & -0.044077 & -0.725523 & -0.851936 \\ \mathrm{Si} & 1.435113 & -.106763 & -1.517828 \\ \mathrm{H} & 1.809599 & -1.165525 & -2.221106 \\ \mathrm{H} & 1.273237 & 1.039744 & -2.656021 \\ \mathrm{H} & 2.593944 & -.286137 & -0.595136 \\ \mathrm{H} & -0.214858 & 2.005865 & 1.357814 \\ \mathrm{C} & -1.959578 & -0.876168 & 0.498461 \\ \mathrm{H} & -2.487104 & -1.039005 & -0.458192 \\ \mathrm{O} & -2.539856 & -0.810444 & 1.574887\end{array}$

Product $\left(\mathbf{R}_{\mathrm{c}}=\mathrm{CHO} ; \mathbf{R}_{\mathrm{a}}=\mathbf{R}_{\mathrm{b}}=\mathbf{R}_{\mathrm{d}}=\mathbf{R}_{\mathrm{e}}=\mathrm{H}\right)$ Energy: -670.79173 hartree

$\begin{array}{lr}\text { O } & 0.866280 \\ \mathrm{C} & 1.012444 \\ \mathrm{H} & 2.067272 \\ \mathrm{C} & 0.214831 \\ \mathrm{H} & 0.142525 \\ \mathrm{H} & 0.689396 \\ \mathrm{C} & -1.159860 \\ \mathrm{O} & -1.569441 \\ \mathrm{Si} & 1.834392 \\ \mathrm{H} & 1.668362 \\ \mathrm{H} & 1.428926 \\ \mathrm{H} & 3.266006 \\ \mathrm{H} & 0.621993 \\ \mathrm{C} & -2.103430 \\ \mathrm{H} & -3.062807 \\ \mathrm{O} & -1.832723\end{array}$

๑. 590696

0.638860

๑. 578180

$-0.493006$

$-0.310833$

$-1.462836$

$-0.547709$

$-1.460224$

$-0.383490$

$-1.826982$

$-0.113679$

$-0.033825$

1. 604508

0.610195

๑. 555799

1. 510588

$-0.842804$

$\odot .576460$

$\odot .876148$

1. 226233

2. 304749

1. 057047

0.619603

$-0.092273$

$-1.798361$

$-1.489181$

$-3.193016$

$-1.592192$

๑. 902117

0.902379

๑. 355558

1. 686048

Reactant ( $\mathbf{R}_{\mathrm{c}}=\mathrm{F} ; \mathbf{R}_{\mathrm{d}}=\mathbf{R}_{\mathrm{e}}=\mathrm{H}$ ) Energy: -542.56394 hartree

$\begin{array}{lll}\text { C } & 0.528860 & 0.691662\end{array}$

$\begin{array}{lll}\mathrm{H} & 0.527569 & 0.706267\end{array}$

$\begin{array}{lll}\mathrm{H} & 1.115627 & 1.426245\end{array}$

C $\quad-0.191702 \quad-0.230057$

F $\quad-0.915535 \quad-1.136218$

$0 \quad-0.340452 \quad-0.438164$

Si $\quad 0.395201 \quad 0.477065$

$\mathrm{H} \quad-0.073514 \quad-0.135604$

$\mathrm{H} \quad-0.031409 \quad 1.892337$

H $\quad 1.869394$

๑. 393489

1.534766

2.614220

1. 002579

0.895322

1.570283

$-0.424034$

$-1.641238$

$-2.897551$

$-1.542209$

$-1.520506$

Transition State $\left(\mathbf{R}_{\mathrm{c}}=\mathrm{F} ; \mathbf{R}_{\mathrm{a}}=\mathbf{R}_{\mathrm{b}}=\mathbf{R}_{\mathrm{d}}=\mathbf{R}_{\mathrm{e}}=\mathrm{H}\right)$

Energy: -656.72692 hartree Imaginary Frequency: $-496 \mathrm{~cm}^{-1}$

$\begin{array}{llll}0 & 0.583459 & -1.387123 & -0.272252\end{array}$

$\begin{array}{llll}C & 0.501734 & -1.423751 & 0.992309\end{array}$

H $\quad 1.426380 \quad-1.431042 \quad 1.575408$

H $\quad-0.380515 \quad-1.861527 \quad 1.473209$

$\begin{array}{llll}C & -0.183556 & 0.531997 & 1.672479\end{array}$

$\begin{array}{llll}\mathrm{H} & 0.301230 & 0.843382 & 2.588955\end{array}$

H $\quad-1.217293 \quad 0.219350 \quad 1.728961$

C $\quad 0.237355 \quad 1.095872 \quad 0.497452$

$\begin{array}{llll}F & 1.343343 & 1.850523 & 0.496819\end{array}$

$0 \quad-0.208606 \quad 0.844286 \quad-0.674786$

$\mathrm{Si} \quad-1.019916-0.628354-1.317008$

$\begin{array}{llll}\mathrm{H} & -2.040700 & 0.216685 & -2.013741\end{array}$

$\begin{array}{llll}H & -0.378391 & -1.342279 & -2.440947\end{array}$

H $\quad-1.853987-1.384335 \quad-0.342231$

Product $\left(\mathbf{R}_{\mathrm{c}}=\mathrm{F} ; \mathbf{R}_{\mathrm{a}}=\mathbf{R}_{\mathrm{b}}=\mathbf{R}_{\mathrm{d}}=\mathrm{R}_{\mathrm{e}}=\mathrm{H}\right)$

Energy: -656.81478 hartree

C $\quad 0.292132-1.067433$

H $\quad 1.346824-1.230516$

H $\quad-0.280859-1.917761$

$\begin{array}{lll}\text { C } & -0.186882 & 0.226965\end{array}$

$\begin{array}{lll}\mathrm{H} & 0.037243 & 0.210214\end{array}$

H $\quad-1.261069 \quad 0.368974$

C $\quad 0.509509 \quad 1.398336$

F $\quad 1.863270 \quad 1.275818$

$0 \quad 0.038355 \quad 2.340990$

Si $\quad-1.227701-1.344928$

$\mathrm{H} \quad-2.335817 \quad-0.417689$

H $\quad-0.911911-1.238780$

H $\quad-1.661028-2.725590$

$-0.701335$

0.715764

0.945527

1. 109133

1. 363862

2.436618

1. 231944

0.741499

0.896077

0.174004

$-1.514847$

$-1.166029$

$-2.953144$

$-1.168980$ 
Reactant $\left(\mathbf{R}_{\mathrm{c}}=\mathrm{NH}_{2} ; \quad \mathbf{R}_{\mathrm{d}}=\mathbf{R}_{\mathrm{e}}=\mathrm{H}\right)$ Energy: -498.73784 hartree C $\quad 0.651582 \quad-0.439005$ $\mathrm{H} \quad 0.533432 \quad-0.427216$

H $\quad 1.644129-0.587918$

C $\quad-0.415446 \quad-0.306618$

$0 \quad-0.350963 \quad-0.303147$

Si $\quad 0.862602 \quad 0.519694$

H $\quad 0.430614 \quad 0.490311$

$\mathrm{H} \quad 0.969897 \quad 1.910922$

$\begin{array}{lll}\mathrm{H} & 2.179452 & -0.151672\end{array}$

$\mathrm{N}-1.731821 \quad-0.099133$

H $\quad-1.925548 \quad-0.469709$

H $\quad-2.394762 \quad-0.447592$
1.599817

2.675281

1.199180

$\odot .796178$

$-0.578524$

$-1.408492$

$-2.822095$

$-0.910866$

$-1.283502$

1. 208134

2. 132211

0.523966

Transition state $\left(\mathbf{R}_{\mathrm{c}}=\mathrm{NH}_{2} ; \mathbf{R}_{\mathrm{a}}=\mathbf{R}_{\mathrm{b}}=\mathbf{R}_{\mathrm{d}}=\mathbf{R}_{\mathrm{e}}=\mathbf{H}\right)$ Energy: -612.90776 hartree Imaginary Frequency: $-445 \mathrm{~cm}^{-1}$

$\begin{array}{llrr}\mathrm{O} & 0.119650 & 1.584306 & -0.202079 \\ \mathrm{C} & 0.374627 & 1.501780 & 1.026337 \\ \mathrm{H} & 1.410121 & 1.420613 & 1.382332 \\ \mathrm{C} & 0.108647 & -0.635083 & 1.652012 \\ \mathrm{H} & -0.331602 & -0.678670 & 2.642473 \\ \mathrm{H} & 1.148743 & -0.921285 & 1.567943 \\ \mathrm{C} & -0.699972 & -0.828984 & 0.556571 \\ \mathrm{O} & -0.236788 & -0.843545 & -0.681022 \\ \mathrm{Si} & 1.092413 & -0.008150 & -1.429917 \\ \mathrm{H} & 1.580942 & -1.193395 & -2.202193 \\ \mathrm{H} & 0.720178 & -.957330 & -2.484257 \\ \mathrm{H} & 2.241299 & -.319993 & -0.548405 \\ \mathrm{H} & -0.350155 & 1.878392 & 1.759014 \\ \mathrm{~N} & -2.058807 & -0.923834 & 0.608680 \\ \mathrm{H} & -2.509687 & -0.774386 & 1.500907 \\ \mathrm{H} & -2.554686 & -0.580023 & -0.204443\end{array}$

Product $\left(\mathrm{R}_{\mathrm{c}}=\mathrm{NH}_{2} ; \quad \mathrm{R}_{\mathrm{a}}=\mathrm{R}_{\mathrm{b}}=\mathrm{R}_{\mathrm{d}}=\mathrm{R}_{\mathrm{e}}=\mathrm{H}\right)$

Energy: -612.99205 hartree

$\begin{array}{lrrr}\mathrm{O} & 0.665729 & -0.236566 & -0.869646 \\ \mathrm{C} & 0.910018 & -0.185211 & 0.547122 \\ \mathrm{H} & 1.834565 & -0.728577 & 0.781022 \\ \mathrm{C} & -0.254163 & -0.824874 & 1.285393 \\ \mathrm{H} & 0.006905 & -0.946848 & 2.339724 \\ \mathrm{H} & -0.433648 & -1.817554 & 0.858237 \\ \mathrm{C} & -1.538031 & -0.010862 & 1.251944 \\ \mathrm{O} & -2.129667 & 0.326199 & 2.276648 \\ \mathrm{Si} & 1.862546 & 0.272764 & -1.928098 \\ \mathrm{H} & 3.093503 & -0.540190 & -1.751439 \\ \mathrm{H} & 1.306326 & 0.087251 & -3.284854 \\ \mathrm{H} & 2.201316 & 1.701784 & -1.699164 \\ \mathrm{H} & 1.037560 & 0.858279 & 0.864256 \\ \mathrm{~N} & -1.994990 & 0.272728 & -0.001842 \\ \mathrm{H} & -1.373625 & 0.158849 & -0.792611 \\ \mathrm{H} & -2.779055 & 0.907829 & -0.071674\end{array}$

Reactant $\left(\mathbf{R}_{\mathrm{c}}=\mathrm{OH} ; \mathbf{R}_{\mathrm{d}}=\mathrm{R}_{\mathrm{e}}=\mathrm{H}\right)$ Energy: - 518.58020 hartree

$\begin{array}{lll}\text { C } & 0.647414 & 0.000000\end{array}$

$\begin{array}{lll}H & 0.522735 & 0.000000\end{array}$

1.640715

2.713390

1. 234056

0.860911

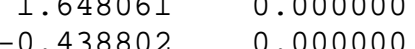

$-0.472075 \quad 0.000000$

$-0.507260$

$\begin{array}{lll}\text { Si } & 0.891707 & 0.000000\end{array}$

H $\quad 1.711025 \quad 1.210968$

$\mathrm{H} \quad 1.711025-1.210968$

$\begin{array}{lll}\mathrm{H} & 0.338597 & 0.000000\end{array}$

$\begin{array}{lll}0 & -1.697947 & 0.000000\end{array}$

$-1.505679$

$-1.267688$

$-1.267688$

$-2.874316$

1. 372209

H $\quad-2.306840$

$\odot .000000$

0.612407

Transition State $\left(\mathbf{R}_{\mathrm{c}}=\mathbf{O H} ; \mathbf{R}_{\mathrm{a}}=\mathrm{R}_{\mathrm{b}}=\mathrm{R}_{\mathrm{d}}=\mathrm{R}_{\mathrm{e}}=\mathrm{H}\right)$

Energy: -632.74977 hartree

Imaginary Frequency: $-463 \mathrm{~cm}^{-1}$

$\begin{array}{lrrr}0 & 0.584790 & -1.425799 & -0.271933 \\ \mathrm{C} & 0.502930 & -1.466024 & 0.985652 \\ \mathrm{H} & 1.419751 & -1.454936 & 1.583740 \\ \mathrm{H} & -0.389197 & -1.877991 & 1.473108 \\ \mathrm{C} & -0.180785 & 0.542481 & 1.694287 \\ \mathrm{H} & 0.321282 & 0.817810 & 2.613036 \\ \mathrm{H} & -1.226569 & 0.272441 & 1.745822 \\ \mathrm{C} & 0.285597 & 1.075131 & 0.523358 \\ \mathrm{O} & -\odot .232562 & 0.852352 & -0.659149 \\ \mathrm{Si} & -1.095927 & -0.551412 & -1.292959 \\ \mathrm{H} & -2.144772 & 0.284917 & -1.958095 \\ \mathrm{H} & -0.461233 & -1.247978 & -2.430979 \\ \mathrm{H} & -1.880120 & -1.335982 & -0.304546 \\ \mathrm{O} & 1.442508 & 1.760818 & 0.505194 \\ \mathrm{H} & 1.699487 & 1.852987 & -0.433346\end{array}$

Product $\left(\mathrm{R}_{\mathrm{c}}=\mathrm{OH} ; \mathrm{R}_{\mathrm{a}}=\mathrm{R}_{\mathrm{b}}=\mathrm{R}_{\mathrm{d}}=\mathrm{R}_{\mathrm{e}}=\mathrm{H}\right)$

Energy: -632.83578 hartree

$\begin{array}{lrrr}\text { O } & 0.229731 & -1.007052 & -0.695837 \\ \mathrm{C} & 0.311938 & -1.105185 & 0.724448 \\ \mathrm{H} & 1.366060 & -1.252539 & 0.967951 \\ \mathrm{H} & -0.252098 & -1.970545 & 1.098764 \\ \mathrm{C} & -0.197718 & 0.175524 & 1.377133 \\ \mathrm{H} & 0.016707 & 0.15080 \odot & 2.451317 \\ \mathrm{H} & -1.273440 & 0.295441 & 1.235300 \\ \mathrm{C} & 0.47680 \odot & 1.368517 & 0.758002 \\ \mathrm{O} & 1.814388 & 1.350582 & 1.002276 \\ \mathrm{O} & -0.066152 & 2.241268 & 0.107553 \\ \mathrm{Si} & -1.186665 & -1.315189 & -1.527906 \\ \mathrm{H} & -2.273833 & -0.363679 & -1.181946 \\ \mathrm{H} & -0.849848 & -1.196284 & -2.961483 \\ \mathrm{H} & -1.669321 & -2.689225 & -1.218509 \\ \mathrm{H} & 2.179229 & 2.127152 & 0.529853\end{array}$


Reactant ( $\mathbf{R}_{\mathrm{c}}=\mathrm{SH} ; \mathbf{R}_{\mathrm{d}}=\mathrm{R}_{\mathrm{e}}=\mathrm{H}$ )

Energy: -841.17733 hartree

$\begin{array}{lll}H & 2.294929 & 0.247770\end{array}$

$\mathrm{H} \quad 2.156910 \quad-0.176108$

C $\quad 0.338426 \quad-0.056039$

$0 \quad-0.556420 \quad-0.270636$

Si $\quad-0.229884$

$\mathrm{H} \quad-1.522649$

$\mathrm{H} \quad 0.242759$

0.071279

$-0.128486$

1.469525

0.790619

$-\odot .428474$

$-1.485565$

$-0.848669$

๑. 006203

$\odot .726387$
0.372510

1.224303

$-0.575947$

0.515578

$-0.498758$

$-2.121702$

$-2.805881$

$-2.259806$

$-2.679734$

2.109679

1.707564

Transition State $\left(\mathbf{R}_{\mathrm{c}}=\mathrm{SH} ; \mathbf{R}_{\mathrm{a}}=\mathbf{R}_{\mathrm{b}}=\mathbf{R}_{\mathrm{d}}=\mathrm{R}_{\mathrm{e}}=\mathrm{H}\right)$ Energy: -955.34086 hartree Imaginary Frequency: $-507 \mathrm{~cm}^{-1}$

$\begin{array}{lr}\text { O } & 0.323038 \\ \text { C } & 0.322575 \\ \text { H } & 1.282995 \\ \text { H } & -0.516735 \\ \text { C } & -0.358618 \\ \text { H } & 0.153933 \\ \text { H } & -1.398507 \\ \text { C } & 0.058124 \\ \text { O } & -0.501256 \\ \text { Si } & -1.363851 \\ \text { H } & -2.441909 \\ \text { H } & -0.802374 \\ \text { H } & -2.123000 \\ \text { S } & 1.532118 \\ \text { H } & 1.718876\end{array}$

$-1.655989$

$-1.639726$

$-1.599774$

$-2.076458$

$\odot .312410$

0.582881

$\odot .018680$

0.855818

0.566518

$-0.903605$

$-0.085621$

$-1.644266$

$-1.650691$

1.814797

1.613716

$-0.297628$

0.969429

1.492574

1.524767

1. 618375

2.535078

1. 702325

0.426553

$-0.716606$

$-1.244621$

$-1.889724$

$-2.395682$

$-0.203625$

0.352853

$-\odot .958947$

Product $\left(\mathbf{R}_{\mathrm{c}}=\mathrm{SH} ; \mathrm{R}_{\mathrm{a}}=\mathrm{R}_{\mathrm{b}}=\mathrm{R}_{\mathrm{d}}=\mathrm{R}_{\mathrm{e}}=\mathrm{H}\right)$

Energy: -955.41872 hartree

$\begin{array}{lrrr}\text { O } & 0.00000 \odot & 0.000 \odot \odot \odot & 0.0000 \odot \odot \\ \mathrm{C} & 0.00000 \odot & 0.00000 \odot & 1.420963 \\ \mathrm{H} & 1.047385 & 0.000 \odot \odot \odot & 1.734641 \\ \mathrm{H} & -0.474211 & -0.904214 & 1.827676 \\ \mathrm{C} & -0.711341 & 1.241069 & 1.962493 \\ \mathrm{H} & -0.575394 & 1.298675 & 3.048594 \\ \mathrm{H} & -1.781158 & 1.201584 & 1.742665 \\ \mathrm{C} & -0.170637 & 2.477890 & 1.286865 \\ \mathrm{O} & -0.724464 & 3.037861 & 0.358983 \\ \mathrm{Si} & -1.312330 & -0.511927 & -0.900648 \\ \mathrm{H} & -2.544912 & 0.263129 & -0.607394 \\ \mathrm{H} & -0.924055 & -0.337886 & -2.314846 \\ \mathrm{H} & -1.600058 & -1.943942 & -0.612251 \\ \mathrm{~S} & 1.402897 & 3.025025 & 1.955224 \\ \mathrm{H} & 1.550616 & 3.995377 & 1.041883\end{array}$

$\odot .0000000$

1.420963

1.734641

1.827676

1.742665

1.286865

0.358983

$-0.900648$

0.607394

0.612251

1.041883

Reactant $\left(\mathbf{R}_{\mathrm{d}}=\mathrm{NH}_{2} ; \mathbf{R}_{\mathrm{c}}=\mathrm{R}_{\mathrm{e}}=\mathrm{H}\right)$ Energy: -498.72145 hartree C $\quad-1.228785 \quad-0.487640$ $\mathrm{H} \quad-1.426374 \quad 0.211020$

C $\quad 0.007723 \quad-0.667691$

$\mathrm{H} \quad 0.190117-1.364582$

$0 \quad 1.150869 \quad-0.044663$

Si $1.238944 \quad 1.091297$

$\mathrm{H} \quad 2.666970 \quad 1.459234$

$\begin{array}{lll}\mathrm{H} & 0.413762 & 2.285714\end{array}$

H $\quad 0.779810 \quad 0.517142$

$\mathrm{N} \quad-2.297503 \quad-1.239230$

$\mathrm{H}-2.760074-1.809122$

$\mathrm{H} \quad-3.007484 \quad-0.613672$

$-0.257658$

0.559394

$-0.739297$

$-1.550917$

$-0.312347$

0.922161

1. 002097

0.610850

2. 212127

$-0.843654$

$-0.137829$

$-1.219878$

Transition State $\left(\mathbf{R}_{\mathrm{d}}=\mathrm{NH}_{2} ; \mathbf{R}_{\mathrm{a}}=\mathbf{R}_{\mathrm{b}}=\mathbf{R}_{\mathrm{c}}=\mathbf{R}_{\mathrm{e}}=\mathrm{H}\right)$ Energy: -612.87713 hartree Imaginary Frequency: $-530 \mathrm{~cm}^{-1}$

$\begin{array}{lrrr}\text { O } & 0.631070 & -1.260632 & -0.661869 \\ \mathrm{C} & 0.496791 & -1.329661 & 0.605156 \\ \mathrm{H} & 1.401954 & -1.397146 & 1.219385 \\ \mathrm{H} & -0.395932 & -1.810524 & 1.030108 \\ \mathrm{C} & -0.185939 & 0.489031 & 1.350309 \\ \mathrm{H} & -1.228513 & 0.223943 & 1.165446 \\ \mathrm{C} & 0.473539 & 1.165814 & 0.345740 \\ \mathrm{H} & 1.398205 & 1.703829 & 0.564623 \\ \mathrm{O} & 0.160836 & 1.052088 & -0.907397 \\ \mathrm{Si} & -0.765215 & -0.248885 & -1.731371 \\ \mathrm{H} & -1.625035 & 0.771970 & -2.420755 \\ \mathrm{H} & -0.128207 & -0.930893 & -2.879830 \\ \mathrm{H} & -1.780963 & -0.991180 & -0.929905 \\ \mathrm{~N} & 0.294765 & 0.654170 & 2.690635 \\ \mathrm{H} & -0.310123 & 1.286545 & 3.212699 \\ \mathrm{H} & 0.276679 & -0.234097 & 3.189873\end{array}$

Product $\left(\mathbf{R}_{\mathrm{d}}=\mathrm{NH}_{2} ; \mathrm{R}_{\mathrm{a}}=\mathrm{R}_{\mathrm{b}}=\mathrm{R}_{\mathrm{c}}=\mathrm{R}_{\mathrm{e}}=\mathrm{H}\right)$ Energy: -612.94680 hartree

$\begin{array}{lll}0 & 0.473949 & -0.854780\end{array}$

$\begin{array}{lll}\text { C } & 0.539536 & -0.921504\end{array}$

$\mathrm{H} \quad 1.592665 \quad-1.028898$

$\begin{array}{lll}H & -0.002266 & -1.798868\end{array}$

$\begin{array}{lll}\text { C } & -0.009605 & 0.348152\end{array}$

$\mathrm{H} \quad-1.074354 \quad 0.469112$

C $\quad 0.732756$

1.525319

1.569917

2.367160

$-1.135420$

$-0.027001$

$-1.302780$

$-2.376793$

0.294239

1.074170

$-0.553480$
-0.936266
0.481308
0.758200
0.866648
1.128398
0.875612
0.537582
0.815098
-0.180537
-1.787985
-1.672598
-3.200260
-1.275011
2.558880
3.037222
2.965408 
Reactant $\left(\mathbf{R}_{\mathrm{e}}=\mathrm{NH}_{2} ; \quad \mathbf{R}_{\mathrm{c}}=\mathbf{R}_{\mathrm{d}}=\mathrm{H}\right)$ Energy: -498.72934 hartree

$\begin{array}{lrrr}\mathrm{C} & 0.047881 & 0.0000 \odot \odot & 1.691512 \\ \mathrm{H} & 0.156390 & 0.0000 \odot \odot & 2.773668 \\ \mathrm{C} & 1.125196 & 0.0000 \odot \odot & 0.896708 \\ \mathrm{H} & 2.136913 & 0.0000 \odot \odot & 1.291761 \\ \mathrm{O} & 1.093178 & 0.000 \odot \odot \odot & -0.458985 \\ \mathrm{Si} & -0.289195 & 0.000 \odot \odot \odot & -1.452314 \\ \mathrm{H} & -1.100632 & -1.238491 & -1.404265 \\ \mathrm{H} & -1.100632 & 1.238491 & -1.404265 \\ \mathrm{H} & 0.385032 & 0.0000 \odot \odot & -2.779526 \\ \mathrm{~N} & -1.235277 & 0.000 \odot \odot \odot & 1.053443 \\ \mathrm{H} & -1.782647 & -0.819655 & 1.311740 \\ \mathrm{H} & -1.782647 & 0.819655 & 1.311740\end{array}$

Transition state $\left(\mathbf{R}_{\mathrm{e}}=\mathrm{NH}_{2} ; \mathbf{R}_{\mathrm{a}}=\mathbf{R}_{\mathrm{b}}=\mathbf{R}_{\mathrm{c}}=\mathbf{R}_{\mathrm{d}}=\mathbf{H}\right)$ Energy: -612.87580 hartree Imaginary Frequency: $-439 \mathrm{~cm}^{-1}$

$\begin{array}{lr}\text { O } & \odot .000000 \\ \mathrm{C} & \odot .000000 \\ \mathrm{H} & \odot .991288 \\ \mathrm{H} & -\odot .765349 \\ \mathrm{C} & -0.929457 \\ \mathrm{H} & -\odot .385942 \\ \mathrm{C} & -0.422479 \\ \mathrm{H} & \odot .587340 \\ \mathrm{O} & -1.004828 \\ \mathrm{Si} & -1.394493 \\ \mathrm{H} & -2.127194 \\ \mathrm{H} & -0.754017 \\ \mathrm{H} & -2.477970 \\ \mathrm{~N} & -2.265226 \\ \mathrm{H} & -2.687608 \\ \mathrm{H} & -2.827289\end{array}$

0.000000

$\odot .00000 \odot$

0.000000

$-0.595970$

1.586743

1.679992

2. 242123

2. 665972

2.185287

0.566715

1.558981

$-0.238421$

$-0.171504$

1.171122

1.448852

1. 493004

Product $\left(\mathrm{R}_{\mathrm{e}}=\mathrm{NH}_{2} ; \quad \mathrm{R}_{\mathrm{a}}=\mathrm{R}_{\mathrm{b}}=\mathrm{R}_{\mathrm{c}}=\mathrm{R}_{\mathrm{d}}=\mathrm{H}\right)$ Energy: -612.95383 hartree

$\begin{array}{lrr}\mathrm{O} & -0.258007 & -0.482159 \\ \mathrm{C} & 0.474383 & -0.846264 \\ \mathrm{H} & 1.523105 & -1.056238 \\ \mathrm{H} & 0.038298 & -1.733534 \\ \mathrm{C} & 0.383166 & 0.325801 \\ \mathrm{H} & 1.007189 & 0.084908 \\ \mathrm{C} & 0.997917 & 1.553238 \\ \mathrm{H} & 1.999300 & 1.408698 \\ \mathrm{O} & 0.457772 & 2.648716 \\ \mathrm{Si} & -0.445111 & -1.580693 \\ \mathrm{H} & -1.248268 & -0.894468 \\ \mathrm{H} & 0.878099 & -1.976438 \\ \mathrm{H} & -1.140820 & -2.805515 \\ \mathrm{~N} & -0.991519 & 0.507481 \\ \mathrm{H} & -1.062303 & 1.403058 \\ \mathrm{H} & -1.553334 & 0.617765\end{array}$
$-0.740407$
0.433496
$\odot .180194$
0.908105
1. 390731
2. 266962
$\odot .738167$
0.280171
0.731105
$-1.990669$
$-3.023320$
$-2.542365$
$-1.516502$
1.819712
2.303217
$\odot .974975$

Transition state

( $\mathbf{R}_{\mathrm{a}}=\mathrm{CHO} ; \mathbf{R}_{\mathrm{c}}=\mathrm{NH}_{2} ; \quad \mathbf{R}_{\mathrm{b}}=\mathrm{R}_{\mathrm{d}}=\mathrm{R}_{\mathrm{e}}=\mathrm{H}$ )

Energy: -725.94348 hartree

Imaginary Frequency: $-449 \mathrm{~cm}^{-1}$

$\begin{array}{lrrr}\text { O } & 0.413197 & 1.186487 & -0.671686 \\ \mathrm{C} & 0.458204 & 1.145337 & 0.586129 \\ \mathrm{H} & 1.412436 & 1.094575 & 1.132812 \\ \mathrm{C} & 0.023661 & -0.949833 & 1.217044 \\ \mathrm{H} & -0.526593 & -0.953748 & 2.151504 \\ \mathrm{H} & 1.071352 & -1.215258 & 1.266416 \\ \mathrm{C} & -0.650783 & -1.187251 & -.036822 \\ \mathrm{O} & -0.048903 & -1.219255 & -1.135181 \\ \mathrm{Si} & 1.452263 & -0.542508 & -1.707180 \\ \mathrm{H} & 1.943152 & -1.798322 & -2.352923 \\ \mathrm{H} & 1.304570 & 0.407026 & -2.825606 \\ \mathrm{H} & 2.466905 & -0.256881 & -0.665557 \\ \mathrm{~N} & -2.011100 & -1.213621 & -0.060314 \\ \mathrm{H} & -2.538533 & -1.554828 & -.731656 \\ \mathrm{H} & -2.382799 & -1.436719 & -0.974490 \\ \mathrm{C} & -0.706405 & 1.679478 & 1.365107 \\ \mathrm{H} & -1.641798 & 1.755626 & \odot .778158 \\ \mathrm{O} & -0.628136 & 2.023093 & 2.538133\end{array}$

Product $\left(\mathrm{R}_{\mathrm{a}}=\mathrm{CHO} ; \mathrm{R}_{\mathrm{c}}=\mathrm{NH}_{2} ; \mathrm{R}_{\mathrm{b}}=\mathrm{R}_{\mathrm{d}}=\mathrm{R}_{\mathrm{e}}=\mathrm{H}\right)$

Energy: -726.02053 hartree

0.8599590 .491955

$\begin{array}{lll}\text { C } & 0.864348 & 0.456418\end{array}$

$-0.881140$

0.380424

1.895790

$-0.682032$

$-0.139388$

$-0.487292$

$\odot .563264$

$-1.627043$

$-0.832917$

$-1.277031$

$-1.561296$

$-0.699181$

$-1.940851$

$-0.928922$

$-\odot .198341$

$\odot .536512$

0.924859

1.117044

2. 180787

1. 014838

0.357127

$-0.632570$

$-1.881476$

$-1.121164$

- 2.998436

$-2.425526$

0.853072

2.7944

$-0.118972$

1. 528525

$-2.162976$

๑. 616103

$-3.163854$

$-0.079193$

1. 824632

○. 287545

0.946048

0.728124

2. 654674

๑. 323511

$-\odot .421061$

2. 023736 


\begin{tabular}{|c|c|c|c|}
\hline \multicolumn{4}{|c|}{ Transition State $\left(T_{S 1}\right) \quad\left(R_{a}=R_{b}=F\right.$} \\
\hline \multicolumn{4}{|c|}{$\begin{array}{l}\text { Energy: }-557.62859 \text { hartree } \\
\text { Imaqinary Frequency: }-751 \mathrm{~cm}^{-1}\end{array}$} \\
\hline 0 & -0.612355 & -0.832998 & 2.004931 \\
\hline C & -0.768023 & 0.403750 & 1.631028 \\
\hline $\mathrm{H}$ & -1.077026 & 1.165436 & 2.385069 \\
\hline $\mathrm{H}$ & -1.351783 & $\odot .625698$ & $\odot .695828$ \\
\hline c & 0.784459 & 0.938261 & 1.008849 \\
\hline $\mathrm{H}$ & 1.431555 & 1.113024 & 1.863292 \\
\hline $\mathrm{H}$ & $\odot .691704$ & 1.783019 & $\odot .330927$ \\
\hline c & $\odot .964162$ & -0.343795 & $\odot .427606$ \\
\hline $\mathrm{H}$ & 1.453692 & -1.152954 & 0.954819 \\
\hline 0 & 0.603736 & $-\odot .670259$ & $-\odot .778814$ \\
\hline Si & $-\odot .369722$ & 0.116883 & -1.968839 \\
\hline H & $-\odot .34495 \odot$ & 1.580822 & -1.784714 \\
\hline H & 0.284286 & $-\odot .270135$ & -3.232706 \\
\hline $\mathrm{H}$ & -1.726009 & $-\odot .444517$ & -1.862601 \\
\hline \multicolumn{4}{|c|}{ Intermediate (oxetane) $\quad\left(R_{a}=R_{b}=R_{c}=R_{d}=R_{e}=H\right)$} \\
\hline \multicolumn{4}{|c|}{ Energy: -557.74468 hartree } \\
\hline 0 & 1.235519 & -0.440197 & 0.033075 \\
\hline C & 1.153524 & -0.520503 & 1.488377 \\
\hline $\mathrm{H}$ & 2.151812 & $-\odot .515753$ & 1.933687 \\
\hline $\mathrm{H}$ & $\odot .611264$ & -1.415446 & 1.805518 \\
\hline C & 0.394783 & 0.807077 & 1.556526 \\
\hline H & $\odot .877465$ & 1.611438 & 2.113843 \\
\hline $\mathrm{H}$ & $-\odot .653832$ & $\odot .732067$ & 1.847203 \\
\hline C & $\odot .582287$ & $\odot .848594$ & $\odot .035146$ \\
\hline H & 1.240334 & 1.631719 & $-\odot .356199$ \\
\hline 0 & -0.580152 & 0.843420 & -0.736804 \\
\hline Si & -1.263846 & $-\odot .638776$ & -1.163587 \\
\hline $\mathrm{H}$ & -2.573228 & $-\odot .253599$ & -1.738587 \\
\hline H & -0.488998 & -1.377430 & -2.185638 \\
\hline $\mathrm{H}$ & -1.497475 & -1.506930 & 0.019928 \\
\hline \multirow{3}{*}{\multicolumn{4}{|c|}{$\begin{array}{l}\text { Transition State }\left(\mathrm{TS}_{\mathrm{S} 2}\right) \quad\left(\mathrm{R}_{\mathrm{a}}=\mathrm{R}_{\mathrm{b}}=\mathrm{R}_{\mathrm{c}}=\mathrm{R}_{\mathrm{d}}=\mathrm{R}_{\mathrm{e}}=\mathrm{H}\right) \\
\text { Energy: -557.69922 hartree } \\
\text { Imaqinary Frequency: }-468 \mathrm{~cm}^{-1}\end{array}$}} \\
\hline & & & \\
\hline & & & \\
\hline 0 & 0.870718 & $-\Theta .465310$ & $-\odot .05 \odot 927$ \\
\hline C & $\odot .7 \odot 3899$ & $-\odot .491355$ & 1.420028 \\
\hline H & 1.697327 & -0.589965 & 1.857286 \\
\hline H & $\odot .077673$ & -1.331875 & 1.728209 \\
\hline C & 0.104201 & 0.907456 & 1.498930 \\
\hline H & 0.618104 & 1.599221 & 2.169811 \\
\hline H & $-\odot .968739$ & $\odot .928564$ & 1.695683 \\
\hline C & 0.373679 & 1.153075 & $-\odot .0 \odot 4571$ \\
\hline H & 1.274065 & 1.742551 & $-\odot .236251$ \\
\hline 0 & $-\odot .580310$ & 1.130614 & $-\odot .868998$ \\
\hline Si & $-\odot .657605$ & -0.933188 & -0.986625 \\
\hline $\mathrm{H}$ & -1.922953 & $-\odot .899395$ & $-\odot .213807$ \\
\hline $\mathrm{H}$ & -0.708173 & -0.738670 & -2.448560 \\
\hline H & $-\odot .274769$ & -2.383286 & -0.866537 \\
\hline
\end{tabular}


Reactant $\left(\mathrm{R}_{\mathrm{c}}=\mathrm{R}_{\mathrm{d}}=\mathrm{R}_{\mathrm{e}}=\mathrm{H}\right.$; $\left.\mathrm{Si}\left(\mathrm{CH}_{3}\right)_{3}\right)$ Energy: -561.09828 hartree

$\begin{array}{llll}\mathrm{H} & 0.400664 & 0.000093 & -3.671916\end{array}$

$\begin{array}{llll}H & 1.321156 & 0.000015 & -2.066259\end{array}$

$\begin{array}{llll}\text { C } & -0.814344 & -0.000163 & -1.974460\end{array}$

$\begin{array}{llll}\mathrm{H} & -1.741075 & -0.000202 & -2.543160\end{array}$

$\begin{array}{llll}0 & -1.067272 & -0.000305 & -0.633434\end{array}$

$\begin{array}{llll}\text { Si } & 0.036515 & 0.000016 & 0.657062\end{array}$

C $\quad-1.068833-0.000282$

$\begin{array}{lll}\text { H } & -0.479201 & -0.000071\end{array}$

$\begin{array}{lll}\mathrm{H} & -1.712804 & 0.882934\end{array}$

H $\quad-1.712256-0.883896$

C $\quad 1.094317 \quad-1.547806$

$\mathrm{H} \quad 1.732212 \quad-1.593873$

H $\quad 0.466828 \quad-2.443496$

$\begin{array}{lll}\mathrm{H} & \mathbf{1 . 7 4 2 7 3 5} & -1.591387\end{array}$

C $\quad 1.093434 \quad 1.548436$

$\begin{array}{lll}\mathrm{H} & 1.741571 & 1.592461\end{array}$

H $\quad 0.465438 \quad 2.443772$

H $\quad 1.731554 \quad 1.594771$

Transition State

$\left(R_{\mathrm{a}}=\mathrm{R}_{\mathrm{b}}=\mathrm{R}_{\mathrm{c}}=\mathrm{R}_{\mathrm{d}}=\mathrm{R}_{\mathrm{e}}=\mathrm{H}\right.$; $\left.\mathrm{Si}\left(\mathrm{CH}_{3}\right)_{3}\right)$

Energy: -675.24374 hartree Imaginary Frequency: $-549 \mathrm{~cm}^{-1}$

$\begin{array}{lrrr}\text { O } & -1.278665 & -0.466949 & 0.722962 \\ \mathrm{C} & -1.177736 & 0.031519 & 1.899582 \\ \mathrm{H} & -1.398756 & -0.626662 & 2.749690 \\ \mathrm{H} & -1.463992 & 1.079153 & 2.071481 \\ \mathrm{C} & 0.734085 & 0.459315 & 2.276633 \\ \mathrm{H} & 0.915793 & 0.509360 & 3.345826 \\ \mathrm{H} & 0.714054 & 1.408733 & 1.756435 \\ \mathrm{C} & 1.202584 & -0.645520 & 1.589184 \\ \mathrm{H} & 1.561848 & -1.533155 & 2.117294 \\ \mathrm{O} & 1.036771 & -0.802324 & 0.321887 \\ \mathrm{Si} & -0.041954 & 0.049731 & -0.887883 \\ \mathrm{C} & 1.443310 & 0.203511 & -2.069466 \\ \mathrm{H} & 1.850311 & -0.778833 & -2.329205 \\ \mathrm{H} & 1.148917 & 0.692949 & -3.004941 \\ \mathrm{H} & 2.259743 & 0.790567 & -1.635522 \\ \mathrm{C} & -0.503872 & 1.865482 & -0.658836 \\ \mathrm{H} & -0.705629 & 2.269573 & -1.657475 \\ \mathrm{H} & -1.386860 & 2.038881 & -0.041962 \\ \mathrm{H} & 0.331602 & 2.451631 & -0.261881 \\ \mathrm{C} & -1.202517 & -1.066024 & -1.852252 \\ \mathrm{H} & -1.088696 & -0.897270 & -2.926721 \\ \mathrm{H} & -0.945348 & -2.111363 & -1.652852 \\ \mathrm{H} & -2.245599 & -0.925318 & -1.567663\end{array}$

\begin{tabular}{|c|c|c|c|}
\hline \multicolumn{4}{|c|}{$\begin{array}{l}\text { Product }\left(\mathrm{R}_{\mathrm{a}}=\mathrm{R}_{\mathrm{b}}=\mathrm{R}_{\mathrm{c}}=\mathrm{R}_{\mathrm{d}}=\mathrm{R}_{\mathrm{e}}=\mathrm{H} ; \mathrm{Si}\left(\mathrm{CH}_{3}\right)_{3}\right) \\
\text { Energy: }-675.32411 \text { hartree }\end{array}$} \\
\hline 0 & $-\odot .067017$ & -0.354212 & -0.177826 \\
\hline C & -1.203268 & ๑. 090861 & -0.911480 \\
\hline $\mathrm{H}$ & -1.324439 & 1.180204 & $-\odot .816511$ \\
\hline $\mathrm{H}$ & -2.121704 & $-\odot .380762$ & -0.538862 \\
\hline C & -0.966911 & $-\odot .278198$ & -2.362163 \\
\hline $\mathrm{H}$ & -1.803420 & ๑. 078934 & -2.979651 \\
\hline H & -0.887181 & -1.361180 & -2.482584 \\
\hline C & 0.305497 & 0.355326 & -2.864606 \\
\hline $\mathrm{H}$ & $\odot .470965$ & 1.401949 & -2.530561 \\
\hline 0 & 1.107392 & -0.196429 & -3.601555 \\
\hline Si & $\odot .141491$ & $-\odot .014152$ & 1.461834 \\
\hline C & -1.293608 & -0.712701 & 2.450766 \\
\hline $\mathrm{H}$ & -1.131842 & $-\odot .563801$ & 3.523149 \\
\hline $\mathrm{H}$ & -2.242818 & $-\odot .231864$ & 2.195756 \\
\hline $\mathrm{H}$ & -1.404677 & -1.786857 & 2.276886 \\
\hline C & 1.745764 & $-\odot .853656$ & 1.915000 \\
\hline $\mathrm{H}$ & 2.571828 & $-\odot .466667$ & 1.312607 \\
\hline $\mathrm{H}$ & 1.993464 & $-\odot .693923$ & 2.968876 \\
\hline $\mathrm{H}$ & 1.683973 & -1.931406 & 1.743123 \\
\hline C & ๑.253612 & 1.841667 & 1.727020 \\
\hline $\mathrm{H}$ & 0.467725 & 2.066480 & 2.776833 \\
\hline $\mathrm{H}$ & 1.055316 & 2.277317 & 1.123773 \\
\hline $\mathrm{H}$ & -0.677578 & 2.355037 & 1.469313 \\
\hline
\end{tabular}




\begin{tabular}{lrrr}
\multicolumn{4}{l}{ Reactant $\left(\mathbf{R}_{\mathbf{c}}=\mathbf{R}_{\mathbf{d}}=\mathbf{R}_{\mathbf{e}}=\mathbf{H} ; \mathbf{S i}\left(\mathbf{C}_{3} \mathbf{H}_{6}\right)\left(\mathbf{C H}_{3}\right)\right)$} \\
Energy: $\mathbf{- 5 9 9 . 0 6 1 2 9}$ hartree \\
$\mathrm{C}$ & -0.659592 & 0.653938 & -2.586677 \\
$\mathrm{H}$ & -0.944087 & 0.832634 & -3.615557 \\
$\mathrm{H}$ & -0.410047 & 1.514528 & -1.977221 \\
$\mathrm{C}$ & -0.654080 & -0.601105 & -2.122813 \\
$\mathrm{H}$ & -0.934910 & -1.442691 & -2.750887 \\
$\mathrm{O}$ & -0.328418 & -1.014404 & -0.859212 \\
$\mathrm{Si}$ & 0.458028 & -0.065351 & 0.306503 \\
$\mathrm{C}$ & -0.675969 & 1.239426 & 1.059309 \\
$\mathrm{H}$ & -0.109713 & 2.022090 & 1.577927 \\
$\mathrm{H}$ & -1.478630 & 1.712038 & 0.489449 \\
$\mathrm{C}$ & 0.104943 & -0.837629 & 1.979213 \\
$\mathrm{H}$ & -0.116620 & -1.901906 & 2.078205 \\
$\mathrm{H}$ & 0.860336 & -0.565756 & 2.725319 \\
$\mathrm{C}$ & -1.119892 & 0.124074 & 2.053083 \\
$\mathrm{H}$ & -1.355987 & 0.481561 & 3.060844 \\
$\mathrm{H}$ & -2.007693 & -0.382847 & 1.663267 \\
$\mathrm{C}$ & 2.184628 & 0.338483 & -0.287962 \\
$\mathrm{H}$ & 2.708345 & 0.965454 & 0.439244 \\
$\mathrm{H}$ & 2.764807 & -0.577669 & -0.429806 \\
$\mathrm{H}$ & 2.158918 & 0.869586 & -1.243041 \\
& & &
\end{tabular}

Transition State

\begin{tabular}{|c|c|c|c|}
\hline & $\begin{array}{l}\mathrm{b}=\mathrm{R}_{\mathrm{c}}=\mathrm{R}_{\mathrm{d}}=\mathrm{R}_{\mathrm{e}}=\mathrm{l} \\
\mathrm{y}:-713.21 \\
\text { inary Frequ }\end{array}$ & $\begin{array}{l}4 \text { hartree } \\
\text { cy: -538 }\end{array}$ & \\
\hline 0 & 1.411406 & -0.979479 & $\odot .309762$ \\
\hline C & 1.380002 & -1.007707 & 1.586173 \\
\hline $\mathrm{H}$ & 2.334917 & -0.947131 & 2.119723 \\
\hline $\mathrm{H}$ & $\odot .6 \odot \odot 593$ & -1.581997 & 2.100080 \\
\hline C & $\odot .506656$ & 0.741734 & 2.268638 \\
\hline $\mathrm{H}$ & $\odot .84369 \odot$ & $\odot .985366$ & 3.271073 \\
\hline $\mathrm{H}$ & -0.492206 & ๑.333498 & 2.181894 \\
\hline C & 1.005315 & 1.479973 & 1.217140 \\
\hline $\mathrm{H}$ & 1.839253 & 2.173288 & 1.354309 \\
\hline 0 & $\odot .680964$ & 1.269098 & $-\odot .017754$ \\
\hline Si & $-\odot . \odot 95925$ & $-\odot .167991$ & -0.799662 \\
\hline C & -1.518495 & -1.063948 & 0.095889 \\
\hline H & -1.846703 & -1.881061 & -0.561866 \\
\hline $\mathrm{H}$ & -1.473038 & -1.469707 & 1.110795 \\
\hline C & -1.674764 & 0.776185 & -1.364505 \\
\hline $\mathrm{H}$ & -1.712072 & 1.865089 & -1.456300 \\
\hline $\mathrm{H}$ & -2.080823 & $\odot .344480$ & -2.288662 \\
\hline$c$ & -2.404164 & $\odot .183421$ & -0.136013 \\
\hline $\mathrm{H}$ & -3.471468 & -0.028062 & $-\odot .271919$ \\
\hline $\mathrm{H}$ & -2.317963 & ๑.868849 & 0.716165 \\
\hline$c$ & $\odot .885770$ & $-\odot .771213$ & -2.280330 \\
\hline $\mathrm{H}$ & $\odot .311342$ & $-\odot .660763$ & -3.202686 \\
\hline $\mathrm{H}$ & 1.803093 & $-\odot .182333$ & -2.373842 \\
\hline $\mathrm{H}$ & 1.183456 & -1.815265 & -2.161508 \\
\hline
\end{tabular}

Product $\left(\mathrm{R}_{\mathrm{a}}=\mathrm{R}_{\mathrm{b}}=\mathrm{R}_{\mathrm{c}}=\mathrm{R}_{\mathrm{d}}=\mathrm{R}_{\mathrm{e}}=\mathrm{H} ; \mathrm{Si}\left(\mathrm{C}_{3} \mathrm{H}_{6}\right)\left(\mathrm{CH}_{3}\right)\right)$ Energy: -713.28629 hartree $\begin{array}{lll}0 & 0.000000 & 0.000000\end{array}$ $\begin{array}{lll}C & 0.000000 & 0.000000\end{array}$ $\begin{array}{lll}\mathrm{H} & 1.031297 & 0.000000\end{array}$ $\begin{array}{lrr}H & -0.503734 & -0.892766\end{array}$ $\begin{array}{lll}\text { C } & -0.719352 & 1.257097\end{array}$ $\begin{array}{lll}\mathrm{H} & -0.707389 & 1.324697\end{array}$ $\begin{array}{lll}\mathrm{H} & -0.707389 & 1.324697 \\ \mathrm{H} & -1.759727 & 1.252019\end{array}$ $\begin{array}{lll}\text { C } & -0.041206 & 2.480839\end{array}$ $\begin{array}{lll}\mathrm{H} & 1.069077 & 2.450666\end{array}$ $\begin{array}{lrr}\mathrm{H} & 1.069077 & 2.450666 \\ 0 & -0.631555 & 3.460338\end{array}$ Si $\quad 0.661263 \quad-1.277803$ $\begin{array}{lll}\text { C } & -0.437232 & -2.812171\end{array}$ $\begin{array}{rr}-0.437232 & -2.812171 \\ 0.117135 & -3.713790\end{array}$ $-1.082836-3.061443$ $-0.045469-1.228715$ $-0.395469-0.293761$ $0.598382-1.739916$ $-1.154637-2.186668$ $-1.513191-2.916411$ $-2.015420-1.599136$ $2.498394-1.412607$ $2.948502-2.186410$ $3.006385-0.466765$ $2.695873-1.678302$

0.000000

1.427203

1.808930

1. 820284

1.871048

2.968254

1. 537356

1. 308467

1.323790

0.881358

$-0.876281$

$-0.845948$

$-1.132024$

- 0.000465

$-2.612778$

$-3.054145$

$-3.337428$

$-2.080304$

$-2.813851$

$-1.747305$

$-0.532340$

$-1.161086$ $-0.741839$

0.510688 
Reactant $\left(\mathrm{R}_{\mathrm{c}}=\mathrm{R}_{\mathrm{d}}=\mathrm{R}_{\mathrm{e}}=\mathrm{H}\right.$; $\left.\mathrm{SiF}_{3}\right)$ Energy: $\mathbf{- 7 4 0 . 8 6 3 9 4}$ hartree $\begin{array}{lll}\text { C } & 0.508794 & -0.034531\end{array}$

$\begin{array}{lll}\mathrm{H} & 0.593841 & 0.254981\end{array}$

$\begin{array}{lll}H & 1.406543 & -0.340923\end{array}$

$\begin{array}{lll}\text { C } & -0.689410 & -0.014806\end{array}$

$\begin{array}{lll}\mathrm{H} & -1.606187 & 0.257029\end{array}$

$\begin{array}{lll}0 & -0.936761 & -0.376495\end{array}$

Si $\quad-0.001166-0.001198$

F $\quad 0.108884 \quad 1.568846$

$\begin{array}{lll}F & -0.677608 & -0.651695\end{array}$

F $\quad 1.479824 \quad-0.566744$

Transition State

$\left(\mathbf{R}_{\mathrm{a}}=\mathbf{R}_{\mathrm{b}}=\mathbf{R}_{\mathrm{c}}=\mathbf{R}_{\mathrm{d}}=\mathrm{R}_{\mathrm{e}}=\mathrm{H}\right.$; $\left.\mathrm{SiF}_{3}\right)$

Energy: -855.03169 hartree Imaginary Frequency: $-465 \mathrm{~cm}^{-1}$

$\begin{array}{lrrr}\text { O } & 1.226151 & -0.534313 & -0.445051 \\ \text { C } & 1.343369 & -0.006232 & -1.586375 \\ \text { H } & 1.664089 & -0.643463 & -2.393430 \\ \text { H } & 1.613184 & 1.032969 & -1.663322 \\ \text { C } & -0.587862 & 0.471898 & -2.288951 \\ \text { H } & -0.630086 & 0.436262 & -3.362153 \\ \text { H } & -0.559201 & 1.444715 & -1.834097 \\ \text { C } & -1.127214 & -0.562994 & -1.577383 \\ \text { H } & -1.466459 & -1.462150 & -2.066411 \\ \text { O } & -1.148717 & -0.603208 & -0.300901 \\ \text { Si } & 0.006134 & 0.014692 & 0.830740 \\ \text { F } & -1.171642 & 0.361151 & 1.858149 \\ \text { F } & 0.467896 & 1.521235 & 0.578183 \\ \text { F } & 0.804119 & -0.919040 & 1.827327\end{array}$

Product $\left(\mathrm{R}_{\mathrm{a}}=\mathrm{R}_{\mathrm{b}}=\mathrm{R}_{\mathrm{c}}=\mathrm{R}_{\mathrm{d}}=\mathrm{R}_{\mathrm{e}}=\mathrm{H} ; \mathrm{SiF}_{3}\right)$ Energy: -855.09087 hartree

$\begin{array}{lrr}\text { O } & -0.062757 & -0.208407 \\ \text { C } & -1.169945 & 0.161113 \\ \text { H } & -1.301911 & 1.236565 \\ \text { H } & -2.067966 & -0.310693 \\ \text { C } & -0.897813 & -0.282700 \\ \text { H } & -1.743775 & 0.004195 \\ \text { H } & -0.791258 & -1.358422 \\ \text { C } & 0.342025 & 0.356925 \\ \text { H } & 0.545644 & 1.383955 \\ \text { O } & 1.050620 & -0.182016 \\ \text { Si } & 0.1430 \odot 2 & -0.023910 \\ \text { F } & -0.987291 & -0.741509 \\ \text { F } & 1.522157 & -0.626052 \\ \text { F } & 0.110548 & 1.488727\end{array}$

$-0.082416$

$-0.885523$

$-0.833695$

$-0.507057$

$-2.309258$

$-2.932149$

$-2.367295$

$-2.893824$

$-2.571481$

$-3.676983$

1.490176

2. 305091

1.900603

1.900533
Reactant $\left(\mathbf{R}_{\mathrm{c}}=\mathbf{R}_{\mathrm{d}}=\mathbf{R}_{\mathrm{e}}=\mathrm{H} ; \mathrm{SiCl}_{3}\right)$ Energy: -1820.82678 hartree

$\begin{array}{lrrr}\text { C } & -2.914416 & 0.0000 \odot \odot & -0.717273 \\ \text { H } & -3.926818 & 0.00000 \odot & -1.072151 \\ \text { H } & -2.762073 & 0.0000 \odot \odot & 0.345797 \\ \text { C } & -1.934714 & 0.00000 \odot & -1.591326 \\ \text { H } & -2.096084 & 0.00000 \odot & -2.650547 \\ \text { O } & -0.599140 & 0.0000 \odot \odot & -1.311442 \\ \text { Si } & 0.330770 & 0.00000 \odot & 0.00000 \odot \\ \text { Cl } & -0.007097 & 1.653934 & 1.136997 \\ \text { Cl } & 2.251964 & 0.00000 \odot & -0.643403 \\ \text { Cl } & -0.007097 & -1.653934 & 1.136997\end{array}$

Transition State

$\left(\mathbf{R}_{\mathrm{a}}=\mathbf{R}_{\mathrm{b}}=\mathbf{R}_{\mathrm{c}}=\mathbf{R}_{\mathrm{d}}=\mathrm{R}_{\mathrm{e}}=\mathrm{H} ; \mathrm{SiCl}_{3}\right)$

Energy: -1934.98437 hartree

Imaginary Frequency: $-445 \mathrm{~cm}^{-1}$

$\begin{array}{lrrr}\text { O } & 1.299630 & 0.038925 & -0.966912 \\ \text { C } & 1.111919 & 0.386848 & -2.167907 \\ \text { H } & 1.703935 & -0.110943 & -2.916663 \\ \text { H } & 0.807771 & 1.396253 & -2.385141 \\ \text { C } & -0.805006 & -\Theta .284118 & -2.777649 \\ \text { H } & -0.807215 & -0.493265 & -3.831631 \\ \text { H } & -1.291142 & 0.617950 & -2.455508 \\ \text { C } & -0.692706 & -1.323948 & -1.905155 \\ \text { H } & -0.477956 & -2.323138 & -2.247713 \\ \text { O } & -0.703776 & -1.183868 & -0.629816 \\ \text { Si } & 0.056695 & -\Theta .036792 & 0.418791 \\ \text { Cl } & -1.456678 & -0.391287 & 1.862212 \\ \text { Cl } & -0.375496 & 1.986935 & 0.136640 \\ \text { Cl } & 1.645045 & -0.541820 & 1.639482\end{array}$

Product $\left(\mathrm{R}_{\mathrm{a}}=\mathrm{R}_{\mathrm{b}}=\mathrm{R}_{\mathrm{c}}=\mathrm{R}_{\mathrm{d}}=\mathrm{R}_{\mathrm{e}}=\mathrm{H} ; \mathrm{SiF}_{3}\right)$

Energy: -1935.05594 hartree

$\begin{array}{lrrr}\text { O } & -0.140015 & -0.224399 & -0.614774 \\ \text { C } & -1.254026 & 0.087025 & -1.437475 \\ \text { H } & -1.431110 & 1.155937 & -1.399726 \\ \text { H } & -2.133601 & -0.419585 & -1.062360 \\ \text { C } & -0.941283 & -0.364234 & -2.850515 \\ \text { H } & -1.791997 & -0.124824 & -3.486896 \\ \text { H } & -0.785833 & -1.434705 & -2.891930 \\ \text { C } & 0.274831 & 0.323449 & -3.430774 \\ \text { H } & 0.428291 & 1.362945 & -3.121117 \\ \text { O } & 1.015112 & -0.193973 & -4.198929 \\ \text { Si } & 0.082191 & -0.027567 & 0.961961 \\ \text { Cl } & -1.343600 & -1.035722 & 2.014827 \\ \text { Cl } & 1.916899 & -0.740374 & 1.429247 \\ \text { Cl } & -0.038848 & 1.947609 & 1.456912\end{array}$

